March 2014

"Cooperative decision-making for the provision of a locally undesirable facility"

Stefan Ambec and Yann Kervinio 


\title{
Cooperative decision-making for the provision of a locally undesirable facility
}

\author{
Stefan Ambec*and Yann Kervinio ${ }^{\dagger \ddagger \S}$
}

March 26, 2014

\begin{abstract}
We consider the decentralized provision of a global public good with local externalities in a spatially explicit model. Communities decide on the location of a facility that benefits everyone but exhibits costs to the host and its neighbors. They share the costs through transfers. We examine the cooperative game associated with this so-called NIMBY ("Not In My Back-Yard") problem. We derive and discuss conditions for core solutions to exist. These conditions are driven by the temptation to exclude groups of neighbors at any potential location. We illustrate the results in different spatial settings. In particular, we construct a hypothetical example on a real administrative unit in which the core is shown to be empty. These results clarify how property rights can affect cooperation and shed further light on a limitation of the Coase theorem.

Keywords: NIMBY, externality, Coase theorem, pollution, waste, core, cooperative game, spatial model.
\end{abstract}

JEL codes: C71, D62, Q53, R53.

\footnotetext{
${ }^{*}$ Toulouse School of Economics, F-31000 Toulouse, France (INRA LERNA)

${ }^{\dagger}$ Toulouse School of Economics, F-31000 Toulouse, France

${ }^{\ddagger}$ AgroParisTech, F-75005 Paris, France

${ }^{\S}$ We wish to thank Olof Johansson-Stenman, Justin Leroux and Vera Zaporozhets for helpful comments. We are especially grateful to Michel Le Breton for his advice and suggestions. This paper also benefited from comments by participants at the 2013 conference of the European Association of Environmental and Resource Economics in Toulouse, the 2013 Public Economic Theory conference in Lisbon and the 2013 Spanish-Italian-Netherland Game theory conference in Vigo. Any remaining error is ours. We acknowledge financial support from ANR (France) through the project ANR-12-BSH1-0003-01 on "Political Economy of the Environment".
} 


\section{Introduction}

The production of activities that are harmful to society is the source of a famous controversy between Arthur Pigou and Ronald Coase. In such a context, Pigou recommended that the producers pay the harmful damages inflicted on third parties. Using the example of the sparks from railway engines that set fire to woods surrounding the tracks, Pigou (1920) argued that the railways should be forced to compensate those whose woods are burnt. In the same example, Coase (1960) challenged the Pigouvian solution. He argued that the parties involved could resolve the problem themselves in the absence of transaction costs, provided the property rights on harmful externalities (or liability rules) were assigned to one of them. Such Coasean bargaining would lead to efficiency, regardless of the allocation of property rights. This is known as the "Coase Theorem".

The Coase theorem was subsequently invalidated in cooperative settings involving more than two players. A famous instance is a version of the Shapley and Shubik garbage game (1969), in which three neighbors decide on where to dump their garbage. For instance, let the disutility of having waste in one's backyard be -1 for one bag of garbage and -2 both for 2 and 3 bags of garbage. When utility is transferable, the efficient outcome is for the three agents to cooperate and to locate the garbage in the backyard of one of them. The total disutility so achieved is -2 . Coasean bargaining predicts that, in the absence of transaction costs, they will exchange garbage and money to reach such a socially optimal outcome. Yet, if a player has the right to dispose of garbage as she or he likes, every group of two players will prefer to dump their garbage into the third player's garden without compensating her or him. In the previous example, one can easily check that whatever way they share the total cost, there will always be a couple of players willing to withdraw and coordinate their dumping on the third player: this game has an empty "core". ${ }^{1}$ More generally, Starrett (1973) pointed out that economies with nonconvexities can have an empty core. Aivazian and Callen (1981) make a similar argument: they provide an example with a polluting facility for which the core in the cooperative game representing Coasean bargaining with a specific liability rule is empty. ${ }^{2}$ Although the above examples do show that the Coase theorem cannot always be demonstrated, they do not tell us in which circumstances the Coase theorem is likely to hold. This paper fills that gap. Using quite a general model of production activities with negative externalities, we investigate what the driving economic parameters are that determine whether the core is empty.

To do so, we deal with a spatial model with externalities. It represents the problem of providing a locally undesirable but globally desirable facility. In Pigou's story on sparks from railway engines, all citizens connected to the railways benefit from it, but those who own wood along the track might suffer from the externality cost. This is the well-known Not In My Backyard (NIMBY) problem. Examples include waste treatment plants, nuclear or coal power utilities, windmills, airports, prisons or, more recently, shale gas wells. ${ }^{3}$ Such facilities are acknowledged to be socially beneficial in the sense of the Hicks-Kaldor

\footnotetext{
${ }^{1}$ The total disutility that such a two-player coalition can guarantee to itself is at least -1: both members drop their garbage on the third player but may still get his or her garbage. Additionally, the disutility of the third agent, is -2 , hence the total disutility is -3 : social efficiency is not achieved. Hence, players may not be able to reach an efficient outcome.

${ }^{2}$ The argument is reproduced by Stearns (1993) with voting instead of bargaining as a collective decision process. In his example, a Condorcet cycle arises in a situation where three communities have to collectively decide where to site a nuclear waste repository.

${ }^{3}$ Some of these projects feature non-excludability of the benefits at the origin of free-riding behaviors; others not. We will emphasize here the garbage game dimension of such problems, which is common to all.
} 
criterion: the social benefits more than offset the social costs. Yet their provision meets strong opposition from neighboring citizens who suffer from negative externalities such as air or water pollution, noise, or amenity losses. ${ }^{4}$ This is why the localization of the facility is a sensitive issue. Some form of compensation should be offered to make it acceptable to the neighboring victims of externality costs. In this paper we examine the decentralized provision of a locally undesirable facility.

The NIMBY problem is first studied in its general form. Several communities plan to build a facility. The benefits from using the facility are excludable and non-rival. The costs are incurred by the host and its neighbors. The communities both agree on location and transfers: they decide on who is going to host the facility and how much the host and its neighbors must be compensated. Coalitions of communities block the agreement if they are better-off building and sharing their own facility (or not building at all). The outside option of coalitions defines a cooperative game associated with the NIMBY problem. Interestingly, the cooperative game exhibits specific properties. It is a cooperative game with externalities in the sense that the welfare that a group of communities can enjoy depends on the cooperative behavior of communities outside the group as well as the localization of the facility they build on their own. Yet externalities in the associated cooperative game can be negative or positive: a group of communities can benefit or suffer from the cooperation of others. Nevertheless, the best that can happen for a coalition of communities is that the other communities are not cooperating. We first define the value function of our cooperative game accordingly: a group of communities do not expect that the others will build their own facility when they oppose an agreement. We thus give maximal incentives for coalitions to deviate and block an agreement. Under some assumptions, we show that only two forces constrain the core: individual rationality and the exclusion of individual communities and communities in the neighborhood of any potential host of the facility. Individual rationality makes sure that all communities benefit from the facility. The motive for exclusion is similar to that in Shapley and Shubik's garbage game: communities are tempted to exclude those who suffer from the negative externality, to avoid compensating them. Thus we restrict considerably the set of inequalities defining the core.

We next propose an index for testing if the core is empty or not. This corresponds to the least-core value of an associated cooperative game. The core is non-empty if and only if such an index is lower than one. We discuss some comparative static properties of the least-core index. We show that the core is less likely to be empty when externality costs increase everywhere except for the optimal location of the facility. Intuitively, this means that the more harmful the project is when located elsewhere than at the optimal location, the easier it is for the communities to reach an agreement. We also show that the problem is exacerbated when the number of communities increases in the linear case. However, the effect of the number of communities is ambiguous in general. Next, we generalize our results for other notions of the core. We finally provide illustrations on different spatial structures, first in the linear case and on simple graphs. Lastly, we compute the index for a French administrative unit to illustrate its potential relevance for real-world NIMBY problems.

\footnotetext{
${ }^{4}$ Richman and Boerner (2006) define a NIMBY as follows "a socially desirable land use that broadly distributes benefits, yet is difficult or impossible to implement because of local opposition".
} 


\section{Related literature}

Most of the theoretical papers in economics on the NIMBY problem rely on a mechanism design approach. A central planner designs a mechanism such as an auction to locate the undesirable facility optimally and to share its cost (O'Sullivan, 1993, Minehart and Neeman, 2002, Perez-Castrillo and Wettstein, 2002, Laurent-Lucchetti and Leroux, 2011). The central planner can impose the mechanism on the communities but does not know their cost. The implemented solution does not guarantee that some communities could not do better by providing the facility by themselves. In contrast, we assume that the cost of hosting the facility is common knowledge and adopt a cooperative approach. Decisions are decentralized to communities that collectively negotiate and can make binding agreements about localization and compensations.

In a cooperative framework, Laurent-Lucchetti and Leroux (2010), Sakai (2012) and Dehez (2013) have analyzed core solutions of cooperative games associated with NIMBY problems. ${ }^{5}$ They all implicitly rely on the assumption that externalities are concentrated within a jurisdiction. In practice, pollution (e.g. air or water pollution, risk of radioactive contamination) spreads out quite widely compared to the size of the communities (e.g. municipalities, countries). To the best of our knowledge, our paper is the first to explicitly introduce spatial externalities in a cooperative framework representing the NIMBY problem. It emphasizes the difficulties that arise when the costs are spread over more than a single community.

Our paper is related to the literature on public good provision which emphasizes the freeriding problem: users can benefit from public goods without contributing to their cost (see e.g. Bergstrom and al., 1986). Free-riding arises when people cannot be excluded from consuming the good. We avoid free-riding by assuming that communities can be excluded from accessing the facility at no cost. However, the potential exclusion of the neighbors of any potential host can still compromise cooperation. Both the NIMBY and public good provision cooperative games are games with externalities. This raises interesting conceptual issues for the definition of the core and the representation of the game in partition form (see e.g. Ray and Vohra, 2001). Such issues are discussed in Section 4.

Spatial dimensions have been introduced for the provision of public and excludable facilities. In Goemans and Skutella (2004), consumers have heterogeneous costs of being connected to the facility and differ on the benefit they enjoy using the facility, depending on its location. They provide conditions for the core to be non-empty. In Le Breton and Weber (2003), both the users and the facility are located along a line. The benefit of using the facility is proportional to the distance between the user and the facility called "transportation" cost. In this model, preferences are single-peaked, in the sense that the closer the facility, the better for the user. This hypothesis plays a crucial role for the existence of core allocations. In contrast, preferences can be single-dipped in the linear representation of our model. Hence, the motive for blocking a global agreement is different: coalitions would like to avoid compensating the neighbors of the community hosting the facility. As a consequence, non-emptiness of the core is no longer guaranteed. ${ }^{6}$

\footnotetext{
${ }^{5}$ Lejano and Davos (2001) also consider coalition formation in the NIMBY problem. In a numerical example, they argue that a compensation scheme that leaves the host indifferent may fail to be a core allocation.

${ }^{6}$ Note that Barberà et al. (2012) and Manjunath (2013) have examined single-dipped preferences for the location of an indivisible bad. They deal with non-transferable utility (no money involved) whereas we assume transferable utility: players can transfer part of their welfare through side-payments. Their focus is on the localization of the public bad with strategy-proof rules. In contrast, we abstract for information
} 
The rest of the paper is organized as follows. Section 2 introduces the general NIMBY cooperative game with excludable benefits. Section 3 presents the main result. In Section 4 we discuss the robustness of this result when externalities are considered in the definition of the cooperative game. Finally, Section 5 provides illustrations of the main result on explicit spatial structures.

\section{The NIMBY cooperative game}

A set $N=\{1, \ldots, n\}(n>1)$ of communities or agents (land owners, municipalities, cities, regions, countries, etc.) might decide to launch a facility such as a waste treatment plant, a utility (nuclear or coal power plant) or a polluting factory. Each community $i \in N$ enjoys an individual benefit $b_{i} \geq 0$ from using the facility. Benefits are non-rival and non-cumulative: once a community has access to a facility, it does not enjoy any additional benefit from accessing a second one. Yet the communities that launch a facility can exclude the others from using it. A facility is therefore a club good: a non-rival and excludable good. A facility generates local nuisances to the host and its immediate neighbors (pollution, risk of accident or contamination, etc.). This is summarized by the matrix of externality costs $\boldsymbol{C}=\left(c_{i j}\right)_{(i, j) \in N^{2}}$ where $c_{i j} \geq 0$ denotes the costs incurred by community $j$ by a facility located at $i$. We call $c_{i i}$ the host cost for every $i \in N$ and $c_{i j}$ the externality cost for any $i \neq j$. Community $j$ is a neighbor of community $i$ if and only if $c_{i j}>0$. The matrix $\boldsymbol{C}$ provides a spatial representation of the problem. Let us denote by $\overline{\mathcal{N}}(i)=\left\{j \in N \mid c_{i j}>0\right\}$ the neighborhood of $i$ including $i$, and by $\mathcal{N}(i)=\left\{j \in N \backslash\{i\} \mid c_{i j}>0\right\}$ the strict neighborhood of $i$. A NIMBY problem $\sigma$ is defined as a triplet $\sigma=(N, \boldsymbol{b}, \boldsymbol{C})$.

Example. Uniform linear NIMBY problems

Throughout the article, we will consider a particular illustrative NIMBY problem: the uniform linear case. A NIMBY is linear if it can be represented by a line in which a link between communities represents externality costs $c_{i j}$. In a line, each community has a neighbor, except the ones at the two ends. If we order communities according to their location from 1 to $n$, it means that the externality costs are $c_{j j+1}>0$ for $j=1$ to $n-1$. A uniform NIMBY problem is characterized by uniform benefits and costs. The benefit per community is denoted by $b$ so that $\boldsymbol{b}=b \boldsymbol{e}$ where $\boldsymbol{e}=(1, \ldots, 1)$. The host cost is $c$ and the externality cost is $\delta c$ for the neighbors of the host, where $\delta$ is a positive parameter reflecting the share of the host's cost that spreads to the neighboring communities. ${ }^{7}$ Uniform linear NIMBY problems are fully characterized by parameters $(n, b, c, \delta)$.

problems so that the public bad can easily be efficiently located. In our setting, localization impacts the value that a deviating coalition can achieve. It thus determines the distribution of the welfare through side-payments.

${ }^{7}$ We insist on the interpretation of $\delta$ as the share of a neighbor's pollution cost as compared to the host's total cost. Formally, the latter is the sum of a technical cost $c_{t}$ (construction, management, etc.) and a pollution cost $c_{p}$. If $\alpha$ denotes the multiplicative change in the pollution cost for the immediate neighbors, the additional cost for each of them is $\alpha c_{p}$. We then get $\delta=\alpha \frac{c_{p}}{c_{t}+c_{p}}$. So $\delta$ captures the decrease of pollution costs with distance, as well as the share of pollution costs in the hosts's total costs. 
The cost matrix of a linear uniform NIMBY problem is:

$$
\boldsymbol{C}=\left(\begin{array}{ccccccc}
c & \delta c & 0 & \cdots & \cdots & \cdots & 0 \\
\delta c & \ddots & \ddots & \ddots & & & \vdots \\
0 & \ddots & \ddots & \ddots & \ddots & & \vdots \\
\vdots & \ddots & \ddots & \ddots & \ddots & \ddots & \vdots \\
\vdots & & \ddots & \ddots & \ddots & \ddots & 0 \\
\vdots & & & \ddots & \ddots & \ddots & \delta c \\
0 & \cdots & \cdots & \cdots & 0 & \delta c & c
\end{array}\right)
$$

The strict neighborhoods are $\stackrel{\circ}{\mathcal{N}}(1)=\{2\}, \stackrel{\circ}{\mathcal{N}}(i)=\{i-1, i+1\}$ for $i=2, \ldots, n-1$ and $\stackrel{\circ}{\mathcal{N}}(n)=\{n-1\}$. In the uniform linear setting, it is efficient to build the facility at one end of the line. The total welfare so achieved is $n b-(1+\delta) c$. Figure 1 provides a spatial representation of a linear uniform problem when the facility is optimally located at one end of the line.

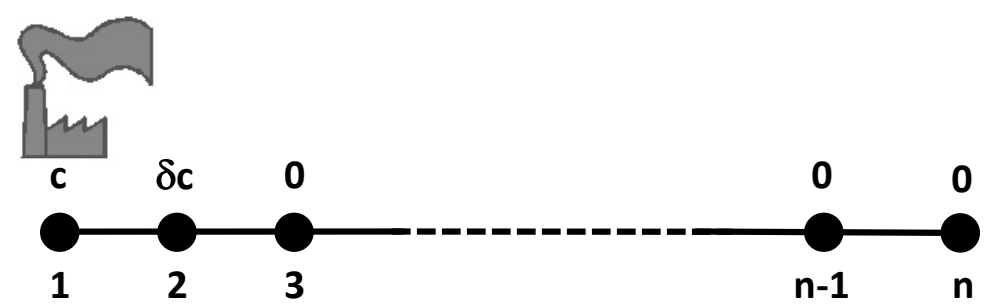

Figure 1: Distribution of the costs at an optimal location in the uniform linear case.

We will use some further notations. For any set of communities $S \subseteq N$ let $b(S)=\sum_{S} b_{i}$ be the total benefit enjoyed by $S$ from running a facility. While the total benefit does not depend on the location of the facility in $S$, total costs do. Let us denote by $c(S)$ the lowest total cost that the members of $S$ incur by building and running a facility. We have $c(S)=\min _{i \in S} \sum_{j \in S} c_{i j}$. A facility should be built if the total benefit exceeds the total cost when located optimally. We assume that $b(N)>c(N)$ : it is efficient to build at least one facility in the grand coalition. Obviously, since a facility is non-rival and benefits are non-cumulative, it is efficient to build only one facility used by all communities in $N$. The total benefit from building a facility optimally located is thus $b(N)-c(N)$. In addition, we assume $b_{i}<c_{i i}$ for every $i \in N$ : it is never efficient for a community to launch a facility alone. Therefore some minimal cooperation of two communities is needed to build a facility. A coalition $S \subset N$ is called a building coalition if $b(S) \geq c(S)$ and a nonbuilding coalition otherwise. We denote by $h \in \operatorname{argmin}_{i \in N} \sum_{j \in N} c_{i j}$ an optimal location in $N$. There may be several optimal locations or hosts $h$ for a given problem $\sigma$. Let $\mathcal{H}$ denote the set of all optimal hosts $\mathcal{H} \equiv \operatorname{argmin}_{i \in N} \sum_{j \in N} c_{i j}$. Finally, we will denote by $\stackrel{\circ}{\mathcal{N}}=\{S \subseteq N \mid \exists i \in N, S \subseteq \stackrel{\circ}{\mathcal{N}}(i)\}$ the set of the subsets of all strict neighborhoods and $\overline{\mathcal{N}}=\stackrel{\circ}{\mathcal{N}} \cup\{\{i\} \mid i \in N\}$.

The communities agree on a location of the facility $h$ and a way to share the net benefit from using it. An allocation is a vector $\boldsymbol{x}=\left(x_{i}\right)_{i=1, \ldots, n}$ where $x_{i}$ denotes community $i$ 's 
benefit with:

$$
\sum_{i \in N} x_{i}=b(N)-c(N)=v(N) .
$$

An allocation of the total net benefit $v(N)$ is induced by budget-balanced transfers $\boldsymbol{t}=$ $\left(t_{i}\right)_{i=1, \ldots, n}$ with $\sum_{i=1}^{n} t_{i}=0$. The host $h$ enjoys a welfare of $x_{h}=b_{h}-c_{h h}+t_{h}$ where $t_{h}$ is the compensation received from hosting the facility. Its neighbors $j$ obtain $x_{j}=b_{j}-c_{h j}+t_{j}$. They are thus paid $t_{j}$ for the nuisances. Other communities $i \in N \backslash \overline{\mathcal{N}}(h)$ get $x_{i}=b_{i}+t_{i}$, thereby paying $-t_{i}$ to finance the compensations $t_{h}+\sum_{j \in \mathcal{N}(h)} t_{j}$.

An allocation is in the core of the NIMBY problem $\sigma$ if it is not blocked by any coalition. We say a coalition $S \subset N$ blocks a distribution of the welfare if its members can achieve a higher welfare by themselves. We need to figure out what a coalition $S$ can achieve by building and running its own facility. It depends on its own behavior and on the behavior of the communities in $N \backslash S$. Indeed, by agreeing to build a facility close to some members of $S$, the communities outside $S$ can exert a negative externality on $S$, hence reducing its value. Technically, the cooperative game induced by the NIMBY problem exhibits externalities: the worth or value of a coalition $S$ depends on the behavior of outside communities. For instance, if the communities outside $S$ agree to build a facility, a member of $S$ who adjoins the facility might suffer from a negative externality and $S$ would experience a welfare loss. We assume that if a coalition $S$ builds a facility, communities outside $S$ do not build any. Such an assumption is in line with the notion of $\gamma$-core whereby communities outside a coalition $S$ play their individual best reply strategies (Chander and Tulkens, 1997). Here, since it is too costly for a single community to build its own facility, its best individual strategy is not to build. Furthermore, it gives higher incentives for a coalition $S$ to block a global agreement since, when doing so, members of $S$ do not anticipate any negative externalities from neighboring facilities. We will discuss this assumption in Section 4.

Under such an assumption, the value or worth of coalition $S \subset N$ is:

$$
v(S)=\max (0, b(S)-c(S))
$$

A core allocation is thus defined as follows:

Definition 1. An allocation $\boldsymbol{x}$ is in the core $\mathcal{C}$ if it satisfies $\sum_{i \in N} x_{i}=v(N)$ and the following core lower bounds:

$$
\forall S \subset N, \sum_{i \in S} x_{i} \geq v(S)
$$

The core is defined by a large number of inequality constraints. Some are binding, others are not. In the next section we show that the problem can be reduced under some assumptions. We propose a simple formula for an index related to the non-emptiness of the core: the least core value.

\section{Existence of core allocations}

We introduce several assumptions which aim at simplifying the problem. The first one is related to the benefit achieved by a coalition formed by excluding a single community or members of a common neighborhood.

Assumption 1. $\forall S \in \overline{\mathcal{N}}, b(N \backslash S) \geq c(N \backslash S)$ 
Assumption 1 implies that coalitions formed by excluding some neighbors of the same community would always build a facility. It holds for local externalities, i.e. when few municipalities are negatively impacted in realtion to the number of beneficiaries. In the uniform linear case, it holds when excluding the neighbors of communities at the extremities of the line $(1$ and $n$ ), or the two neighbors of a middle-community $i$ (with $1<i<n$ ), would not prevent the remaining communities from building a facility. The first requirement is met when $(n-1) b \geq c$ while the second holds true when $(n-2) b \geq c .{ }^{8}$ The last inequality provides a condition on the parameters $n, b$ and $c$ such that Assumption 1 holds in the uniform linear case.

Assumption 2. The optimal host is not unique: $|\mathcal{H}|>1$.

Assumption 2 might appear quite restrictive. Yet it holds in the linear case and can be replaced by a different one for the main result, as discussed in Appendix C.

Relying on the above assumptions, we can significantly reduce the set of lower bounds defining the core.

Proposition 1. Under Assumptions 1 and 2, an allocation $\boldsymbol{x}$ is in the core $\mathcal{C}$ if and only if

$$
\begin{gathered}
\sum_{i \in N} x_{i}=v(N) \\
\forall i \in N, x_{i} \geq 0 \\
\forall i \in N, x_{i} \leq b_{i} \\
\forall S \in \stackrel{\circ}{\mathcal{N}}, \sum_{i \in S} x_{i} \leq b(S)-(c(N)-c(N \backslash S)) .
\end{gathered}
$$

Proposition 1 clarifies what constrains core allocations. Condition (1) is the efficiency condition. Condition (2) captures individual rationality: as we assume any single community anticipates no external cost from its withdrawing, it should be guaranteed 0 in core allocations. In regard to this lower bound on individual allocations, Condition (3) imposes a higher bound on individual allocations: the rationality of the coalitions of size n- 1 ensures that no agent can be subsidized in the grand coalition. From Assumption 2, this requirement also holds for the host. Finally, Condition (4) reflects the possible exclusion of the host's neighbors: by excluding some neighbors of a potential host, a coalition disregards part of the externality costs of the facility. Note that, eventhough it is not taken into account by the remaining coalition, the excluded communities could still suffer from the externality costs. Due to such possibility of costless exclusion, the welfare of the host's neighbors is bounded upward. The point is that the upper bounds should not only hold for the actual host's neighbors, but also for all potential host's neighbors. Therefore, a coalition $S$ of neighbors of a same community should contribute to the project at least to the extent of the cost saved by excluding them, that is, $c(N)-c(N \backslash S)$. We need this

\footnotetext{
${ }^{8}$ In the first case, excluding a community at the extremity of the line allows a cost $\delta c$ to be saved so that the total cost incurred by the coalition which excludes 1 or $n$ is $(1+\delta) c$. Yet the coalition loses the benefit $b$ from the excluded community so that the total benefit is $(n-1) b$. In the second case, by excluding two communities that are neighbors of a middle-community $i$, the coalition can save the two externality costs $2 \delta c$ by locating the facility at $i$, although they loose the benefit of the two neighbors from using the facility so that the total benefit is $(n-2) b$.
} 
condition to hold for every coalition of neighbors of a same community, that is for all coalitions in $\stackrel{\circ}{\mathcal{N}}$.

We note that these constraints can be stringent enough to undermine the existence of core allocations: when exclusion is profitable enough, the allocation of the full value of the facility could be impossible in the grand coalition as such constraints would require the collection of more than the total cost of the project. The understanding we get from Proposition 1 leads us to a general statement about the existence of the core in NIMBY games. The following condition will be imposed.

Assumption 3. $\forall i \in N, b_{i} \geq \max _{j \in N \backslash\{i\}} c_{j i}$

Assumption 3 states that the cost borne by a community when the facility is located at one of its neighbor's never surpasses its own benefit. In the uniform linear case, this means that $b \geq \delta c$ : the externality cost is bounded by the benefit of using the facility. In this specific case, we note that $b \geq \delta c$ is a necessary condition for the core not to be empty as we know that community 2 would have a maximum welfare of $b-\delta c$, which is negative when the condition is not met. This assumption is made in order to focus on cases for which individual rationality is not a source of emptiness of the core. We will see in Section 4 that it can be relaxed when considering more general notions of the core.

The following Proposition provides a simple test for non-emptiness of the core, involving the additive least-core value $I(\boldsymbol{C})$ of the game. Conceptually, the additive least-core value (Maschler et al, 1979) quantifies the necessary decrease in all core lower bounds for the core to be non-empty.

Proposition 2. Under Assumptions 1 to 3, the core is non-empty if and only if $I(\boldsymbol{C}) \leq 1$ where

$$
I(\boldsymbol{C})=\max _{\chi}\left\{\sum_{S \in \overline{\mathcal{N}}} \chi_{S}\left(1-\frac{c(N \backslash S)}{c(N)}\right) \mid \forall i \in N, \sum_{S: i \in S} \chi_{S}=1, \chi_{S} \geq 0\right\}
$$

The proof is provided in Appendix B. A similar result can be obtained with a different assumption than Assumption 2. This is stated and proved in Appendix C. The general scheme of the proof is the following: starting from the result of Proposition 1, we show that individual rationality constraints are never binding in a linear program related to non-emptiness of the core using Assumption 3. The expression of the dual of the resulting linear program then leads to Proposition 2.

Proposition 2 provides us with an index which only depends on the cost structure. The index $I(\boldsymbol{C})$ considers the savings induced by the exclusions of single agents and subsets of strict neighborhoods. It consists in the computation of the extent of the savings induced by such exclusion on all balanced collections of subsets of neighbors. ${ }^{9}$ This is a combinatorial problem which is difficult to solve in general. Yet the computational complexity of $I(\boldsymbol{C})$ is greatly reduced as compared to the general problem of the existence of the core: it restricts the set of constraints to consider from $2^{n}$ to less than $2^{\max _{i \in N}|\overline{\mathcal{N}}(i)|}$. As we will see in the following example and in Section 5, it can be computed for specific spatial structures.

This index also has a natural interpretation. If $I(\boldsymbol{C})$ does not exceed 1 , there exists at least one localization of the facility and an allocation of the net benefit that is immune to

\footnotetext{
${ }^{9} \mathrm{~A}$ collection $\mathcal{B}$ of coalitions is said to be balanced if and only if there exist strictly positive weights $\chi^{\mathcal{B}}=\left(\chi_{S}^{\mathcal{B}}\right)_{S \in \mathcal{B}}$ such that, for any $i \in N, \sum_{S \in \mathcal{N}: i \in S} \chi_{S}^{\mathcal{B}}=1$.
} 
blocking: the provision of the facility can be decentralized in the economy. If $I(\boldsymbol{C})$ exceeds 1 , no such allocation exists. In either case, the index has a quantitative interpretation: it quantifies the maximal decrease in the $\operatorname{costs} c_{i i}$ at all locations $i \in N$ that the problem can sustain. An index of 0.8 indicates that at most $20 \%$ of the total cost in $N$ could be withdrawn to the cost at any location and the core would remain non-empty. An index of 1.2 indicates that at least $20 \%$ of the total cost in $N$ should be added to the cost at any location for the core to be non-empty. ${ }^{10}$

The index $I(\boldsymbol{C})$ allows us to perform comparative statics in the general case of NIMBY games.

Proposition 3. The least core index $I(\boldsymbol{C})$ weakly increases when $\boldsymbol{C}$ decreases while the minimal cost in the grand coalition is unchanged.

The proof of Proposition 3 is developed in Appendix E. It may appear surprising at first sight: when costs decrease everywhere but at the host, the core shrinks. The reason is that a decrease in the costs which leaves the costs at the host unchanged, increases the profitability of the deviation for all coalitions. As a result, $I(\boldsymbol{C})$ weakly increases: the core is more likely to be empty. An illustration will be provided in Section 5.1 for the case of graphs. In the linear case, the number of communities $n$ also causes the leastcore value $I(\boldsymbol{C})$ to increase. However, this result does not generalize to all problems. A counterexample will be provided in Section 5.1.

Example. A first illustration of Proposition 2 can be provided in the uniform linear case. In such problems, an explicit computation of $I(\boldsymbol{C})$ (detailed in Appendix D) leads to a higher bound on the parameter $\delta$ which depends only on the parameter $n$, as stated in the following corollary. ${ }^{11}$

Corollary 1. Under Assumptions 1 and 3, the core of the uniform linear NIMBY problem $(n, b, c, \delta)$ is non-empty if and only if

$$
\delta \leq \bar{\delta}(n)= \begin{cases}\frac{2}{n-2} & \text { if } n=4 k, k \in \mathbb{N} \\ \frac{2}{n-1} & \text { if } n=4 k+1, k \in \mathbb{N} \\ \frac{2}{n} & \text { if } n=4 k+2, k \in \mathbb{N} \\ \frac{2}{n-1} & \text { if } n=4 k+3, k \in \mathbb{N}\end{cases}
$$

Hence, for any number of communities there exists a critical level of $\delta$ above which the core is empty or, for any $\delta$ there exists a critical number of communities above which the core is empty.

\section{A cooperative game with externalities}

The results of the previous section stand for a notion of the core which relies on a coalition's anticipation that outside members will not build any project. In some cases, it may

\footnotetext{
${ }^{10}$ Other meaningful quantities could be built in this context. A recent proposal, the cost of stability (Bachrach et al, 2009), quantifies by how much the cost associated with all coalitions except the grand coalition $N$ should be increased at least so that the core in non-empty. The interpretation of such a value would be appealing in this setting as it would capture the minimal transaction cost associated with the formation of a blocking coalition $S$ - or equivalently the minimal subsidy to the grand coalition - required to stabilize the grand coalition. However, as it does not have an explicit form in this context, we choose to focus on the additive least core value. We thank Michel Le Breton for pointing out this work.

${ }^{11}$ Note that Assumption 2 is always satisfied in the uniform linear case.
} 
be unrealistic to assume such behaviors. For instance, a single community or a small coalition would more likely expect outside members to cooperate on building and sharing a facility. In this section, we examine alternative and plausible expectations on the behavior of outsiders that a coalition might form. In doing so, we generalize the result to other notions of the core.

As discussed above, the cooperative game induced by the NIMBY problem is a cooperative game with externalities: the value of any coalition $S$ depends on the cooperative behavior of communities outside $S$ and their related facility-building decisions. We now formalize the problem in partition form (Thrall and Lucas, 1963). Let $\mathbb{P}(N)$ be the set of all partitions of $N$. The cooperative behavior of communities is summarized by an element $\mathcal{P}$ of $\mathbb{P}(N)$ where each element $S$ of $\mathcal{P}$ is a coalition. The members of $S$ jointly decide on whether to build a facility and on its location. Let us denote $S$ 's building decision by its location choice $l \in S \cup\{0\}$ where $l=0$ if no facility is built. In a partition $\mathcal{P}=\left\{S_{1}, \ldots, S_{m}\right\}$, each coalition of communities $S_{i} \in \mathcal{P}$ picks one of its best location decisions $l_{i}$. A rational location vector in partition $\mathcal{P}$ is a vector $\boldsymbol{l}=\left(l_{1}, \ldots, l_{m}\right)$ where each decision $l_{i}$ minimizes the cost of the facility (the cost of hosting the facility and the externality costs within $S_{i}$ ). Let us denote by $\mathcal{L}(\mathcal{P})$ the set of rational location decision vectors in the partition $\mathcal{P} .{ }^{12}$ They can be multiple due to potential indifference. For instance, in the linear homogeneous NIMBY problem with $n=5$ communities and $2 b<c \leq 3 b$, the partition $\{\{1,2\},\{3,4,5\}\}$ might implement two different location vectors $(0,3)$ and $(0,5)$. They are both rational. Yet $(0,5)$ is preferred to $(0,3)$ by coalition $\{1,2\}$ since in the first case one of its members, namely community 2 , will incur the negative externality cost $\delta c$.

In a standard approach, the value function of a game with externalities depends on the coalition $S$ and the partition in which the coalition is embedded. In the NIMBY game, it also depends on the rational location vector. Hence, we define the value in partition function form $v$ as a function that assigns to every coalition $S$, partition $\mathcal{P}$ of $N$, and rational location vector $\boldsymbol{l} \in \mathcal{L}(\mathcal{P})$, a real number $v(S, \mathcal{P}, \boldsymbol{l})$. It is the welfare achieved by coalition $S$ embedded in the partition $\mathcal{P}$ with the rational location vector $\boldsymbol{l}$ on $\mathcal{P}$. The value is then defined for any potential configuration in terms of partition and rational location decisions. Yet some of those configurations might still appear irrelevant because they are unlikely. We exclude those configurations by introducing the notion of restrictions. A restriction $R$ is a mapping which assigns to each coalition $S$ a doublet $R(S) \in \mathbb{P}(N) \times \mathcal{L}(\mathcal{P})$. Such a function can be interpreted as the expectations of a deviating coalition regarding the behavior of outside members. The value of $S$ under a restriction $R$ is denoted $v^{R}(S)=$ $v(S, R(S))$. Expectations are taken as given here but they could be endogenized following the literature on dynamic coalition formation (see e.g. Chwe, 1995; Bloch, 1996; Ray and Vohra, 1997; Yi, 1997).

We now define the core based on restriction functions. A coalition $S$ in a partition $\mathcal{P}$ blocks a global agreement $\boldsymbol{x}$ under restriction $R$ if it can achieve a higher welfare under such a restriction. An allocation belongs to the $R$-core of the cooperative game associated with the NIMBY problem, denoted $\mathcal{C}^{R}$, if it is not blocked by any coalition of $N$. Formally:

\footnotetext{
${ }^{12}$ Two comments are called for here. First, our restricting the attention to the set of rational decisions is in contrast with the standard approach of the $\alpha$-core and $\beta$-core which respectively consider what a coalition can achieve regardless of the behavior of outside members or when having the possibility to adjust to others actions. Consistently with a remark by Laffont (1977) in the context of the garbage game, the $\alpha$-core would never be empty in our context. Second, in our case, location decisions are independent. Yet, in the case of non-excludable facilities, strategic interactions would arise among coalitions for the provision of facilities.
} 
Definition 2. Let $R$ be a restriction. An agreement $\boldsymbol{x}$ is in the $R$-core $\mathcal{C}^{R}$ if it satisfies $\sum_{i \in N} x_{i}=v(N)$ and the following core lower bounds:

$$
\forall S \subset N, \sum_{i \in S} x_{i} \geq v^{R}(S)
$$

As an illustration, we propose to discuss two specific restrictions relying on polar assumptions on the behavior of outside members.

We call the first restriction Collapse In Outside Cooperation (CIOC). When deviating from a global agreement by blocking an allocation, a coalition $S$ expects that the remaining communities will not cooperate to build facilities. It is formally defined by: $\forall S \subset N$, $R(S)=\left(\left\{S,\{i\}_{i \in N \backslash S}\right\}, \boldsymbol{l}\right)$. Note that since communities outside $S$ are singletons and that we assume that no community would build on its own, they never build. The location decision vector $\boldsymbol{l}$ boils down either to no-building at all, or to a single facility located inside $S$. If $S$ builds a facility, there might be multiple optimal localizations of the facility in $S$. Yet, all these localizations lead to a single value $v^{c}(S)=b(S)-c(S)$. We then have $v^{c}(S)=\max (0, b(S)-c(S))$. This is the notion of the core that we have examined in the previous sections. It corresponds to the notion of the $\gamma$-core introduced in the context of public good games (see e.g. Chander and Tulkens, 1997).

The second restriction we consider corresponds to the delta assumption of full cooperation and its associated notion, the $\delta$-core. We call this second restriction Rational Hostile Outside Cooperation (RHOC). It is formally defined by $\forall S \subset N, R(S)=(\{S, N \backslash S\}, \boldsymbol{l})$. The expectations of a coalition $S$ when considering blocking an allocation is that all the other players will cooperate and (potentially) build a facility. Moreover, coalition $S$ expects that if the coalition $N \backslash S$ is indifferent between different locations, it will locate it at the worst place from $S$ 's point of view. Formally, the RHOC value function for a coalition $S$ is defined as $v^{r}(S)=\min _{\boldsymbol{l} \in \mathcal{L}(\{S, N \backslash S\})} v(S,\{S, N \backslash S\}, \boldsymbol{l})$.

We first investigate the cooperative externalities in the NIMBY problem. A cooperative game exhibits positive (resp. negative) externalities if coalitions benefit (resp. suffer) from the cooperative behavior of players outside (De Clippel and Serrano, 2008). It turns out that externalities in the cooperative game induced by the NIMBY problem can be either positive or negative. The following proposition links the value functions of the game in partition form, the CIOC and the RHOC restrictions.

Proposition 4. For any $S \subset N, \mathcal{P} \ni S$ and $\boldsymbol{l} \in \mathcal{L}(\mathcal{P})$,

$$
v^{c}(S) \geq v(S, \mathcal{P}, \boldsymbol{l})
$$

We might have $v^{r}(S)>v(S, \mathcal{P}, \boldsymbol{l})$ or $v^{r}(S)<v(S, \mathcal{P}, \boldsymbol{l})$ depending on $\mathcal{P}$

We might have $v(S, \mathcal{P}, \boldsymbol{l})>v\left(S, \mathcal{P}^{\prime}, \boldsymbol{l}\right)$ or $v(S, \mathcal{P}, \boldsymbol{l})<v\left(S, \mathcal{P}^{\prime}, \boldsymbol{l}\right)$ when $\mathcal{P}^{\prime}$ is a finer partition of $N$ including $S$.

First, the CIOC value is the highest possible value that a coalition can obtain by deviating from the global agreement. This is because a coalition can only be bothered by nuisances generated by the facilities built by outsiders. So the best that can happen for a coalition is that the outsiders do not build any facility which holds under CIOC.

Second, the RHOC value can be lower or higher than the value with other partitions. This can be shown by example in the 5-player uniform linear NIMBY problem. For $2 b \geq c$ the 
lowest value for coalition $S=\{2\}$ would be achieved with $\mathcal{P}=\{\{2\} ;\{1,4\} ;\{3,5\}\}$ and location decisions $(0,1,3)$ because $S$ would undergo the externalities linked to 2 facilities instead of a single one in the case $\mathcal{P}=\{\{2\} ;\{1,4,3,5\}\}$. This remark emphasizes the fact that full cooperation of outsiders is not the worst that can happen to a coalition.

Third, a coalition does not necessarily benefit from the merger of other coalitions. For instance, $S$ could experience a negative externality when two former non-building coalitions merge and build next to it. This would be the case in the homogeneous linear case with 5 communities when $c \leq 4 b<2 c$ and $\mathcal{P}=\{\{2\} ;\{1,4\} ;\{3,5\}\}$. The merger to $\mathcal{P}=$ $\{\{2\} ;\{1,4,3,5\}\}$ would induce the construction of a facility at 1 and make the worth of $\{2\}$ decrease.

As a consequence of Proposition 4, we know that for any restriction $R, \mathcal{C}^{c} \subseteq \mathcal{C}^{R}$. The CIOCcore is the most restrictive notion of a core as it amounts to considering that coalitions do not take into account the negative externality that outside members could exert on them. Hence, the emptiness of the CIOC-core does not imply the emptiness of any restricted core. This leads us to question the generality of Proposition 2, and more especially to doubt whether the necessary character of the identified condition would extend to any restricted core. We actually show that this condition carries forward to any restriction, provided we additionally assume that neighborhoods are small enough so that they never build when excluded. This is the sense of Assumption 4.

Assumption 4. $\forall S \in \overline{\mathcal{N}}, b(S)<c(S)$

Assumption 4 suffices for any coalition of neighbors of a same community not to build a facility on their own. ${ }^{13}$ It holds when the number of direct neighbors is limited as compared to the minimal number of communities for which building a facility is efficient. For instance, it does hold in the uniform linear case when a minimum of three communities is needed to build a facility, because each community has at most two neighbors. Formally, it requires that $2 b<c$, so that no neighborhood of a community would build on its own. ${ }^{14}$

Assumption 3 will also be needed. Yet, it can be weakened to the following assumption.

Assumption 5. $\forall S \in \overline{\mathcal{N}}, b(S) \geq \sum_{i \in S} \max _{j \in N \backslash\{i\}} c_{j i}+v^{R}(S)$

Unfortunately, Assumption 5 has no direct interpretation. Yet, along with Assumptions 1, 2 and 4, it generalizes Proposition 2 to any notion of a restricted core.

Proposition 5. Under Assumptions 1, 2, 4 and 5, for any restriction $R$, the $R$-core is non-empty if and only if $I(\boldsymbol{C}) \leq 1$.

Under Assumption 2, we can show that core allocations are constrained by two classes of coalition. First, large coalitions consisting in the exclusion of neighborhoods still have an incentive to exclude. They are building coalitions from Assumption 1 and their value does not depend on the behavior of outside members, as Assumption 4 guarantees that no subset of a neighborhood would build a project. Second, the rationality of non-building coalitions can no longer be restricted to individual rationality. However, Assumption 5 is sufficient

\footnotetext{
${ }^{13}$ Along with Assumption 1, it emphasizes a crucial feature for our results to hold: the size asymmetry between neighborhoods and their complementary should be sufficient to induce different building decisions. For this reason, our results apply to local pollution at the scale of $N$.

${ }^{14}$ If two communities neighboring a community $i$ with $1<i<n$ share a facility, they incur the hosting cost but no externality cost for a benefit of $2 b$.
} 
for these constraints to be non-binding in a linear program related to the emptiness of the core. Hence, the results discussed earlier are robust to alternative specifications of the behavior of outside members.

Before moving to the illustrative examples, we briefly discuss the case of non-excludable benefits. It includes for instance NIMBY problems such as shale gas wells or nuclear waste repositories. When benefits are non-excludable, free-riding compromises the existence of core allocations in addition to exclusion: coalitions of communities are tempted to block a global agreement because they can benefit from the facility without paying its cost. However, deviating coalitions may be tempered by two forces. First, large coalitions would not rationally expect remaining communities to build a project by themselves. Hence, free-riding would affect the core lower bounds only for small coalitions. Second, a small deviating coalition may expect the project to be located at its borders when withdrawing from the grand coalition as its interests would no longer be taken into account. At first sight, it would mitigate free-riding incentives. Free-riding never occurs under the CIOC hypothesis as it requires that any deviating coalition anticipate that outside members would not build a project. The RHOC core provides us with an interesting insight on this problem as it presupposes cooperation among remaining communities and the associated credible threat. Therefore, we focus on the RHOC-core. We focus on the linear case when benefits are non-excludable.

Example. Let us consider the uniform linear case with $n \geq 6$. Under Assumptions 1 and 4, the RHOC-core of a uniform linear NIMBY problem with at least six communities and non-excludable benefits is empty. Indeed, for any RHOC-core allocation $\boldsymbol{x}$, the core lower bound of the coalition $N \backslash\{2\}$ (respectively $N \backslash\{n-1\}$ ) requires $x_{2} \leq b_{2}-\delta c$ (resp. $\left.x_{n-1} \leq b_{n-1}-\delta c\right)$. On the other hand, we know that the coalition $N \backslash\{2, n-1\}$ is a building coalition due to Assumption 4. Hence the coalition $\{2, n-1\}$ can free-ride and its core lower bound is written $x_{1}+x_{n-1} \geq b_{1}+b_{n-1}-\delta c$. The latter condition is not compatible with the other two conditions identified. Hence the RHOC-core is empty.

We conclude this part by insisting that the emptiness of the core in the non-excludable case is likely to stem from the interplay between free-riding incentives and the garbagegame dimension of the problem. The latter dimension puts a higher bound on the welfare of the neighborhoods. When benefits are excludable, these small coalitions will often not get more in a core allocation than what they would achieve if they withdrew, even if they have to bear the threat imposed by the remaining communities. Therefore, even if credible threats exist, they would often fail to stabilize the grand coalition. In the following section we redirect the focus to the CIOC-core $\mathcal{C}$ in explicit spatial structures with excludable benefits.

\section{$5 \quad$ Illustrations on explicit spatial structures}

\subsection{Uniform NIMBY problems on graphs}

The linear example developed previously put the emphasis on the effect of the number of individuals on the core. We propose here a natural extension of the linear case which allows us to investigate the effect of the spatial structure on the core. For a given NIMBY problem $(N, \boldsymbol{b}, \boldsymbol{C})$, we restrict our attention to NIMBY problems on graphs. As in the linear case, the cost of building the project at $i$ is the same for all. However, it entails 
an identical additional cost $\delta c$ on each of $i$ 's neighbors. Hence, the matrix of costs can be written $\boldsymbol{C}=c I_{n}+\delta c G$ where $I_{n}$ is the identity matrix and $G$ is the adjacency matrix of a simple graph (with values 0 on the diagonal). A NIMBY problem on a graph is fully characterized by parameters $(N, \boldsymbol{b}, c, \delta, G)$.

Example. Figure 2 below represents the graph associated with the following cost matrix for $n=6$ :

$$
\boldsymbol{C}=\left(\begin{array}{cccccc}
c & \delta c & \delta c & 0 & 0 & 0 \\
\delta c & c & \delta c & 0 & 0 & 0 \\
\delta c & \delta c & c & \delta c & 0 & 0 \\
0 & 0 & \delta c & c & \delta c & \delta c \\
0 & 0 & 0 & \delta c & c & \delta c \\
0 & 0 & 0 & \delta c & \delta c & c
\end{array}\right)
$$

On this graph, a facility built at 1 would yield an external cost $\delta c$ at 2 and 3 . The minimal cost in the grand coalition is $c(N)=c+2 \delta c$. The efficient locations are $1,2,5$ and 6 .

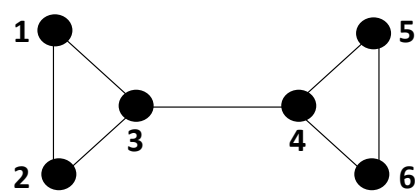

Figure 2: A graph with 6 communities

As in the linear case, the condition for non-emptiness can be stated as an upper bound on the parameter $\delta$.

Corollary 2. Under Assumptions 1, 2 and 3, the core of a NIMBY problem on a graph $(N, \boldsymbol{b}, c, \delta, \boldsymbol{G})$ is non-empty if and only if $\delta \leq \bar{\delta}(\boldsymbol{G})$ where $\bar{\delta}(\boldsymbol{G})>0$.

The proof and an explicit expression for the critical value $\bar{\delta}(\boldsymbol{G})$ are provided in Appendix $\mathrm{G}$, which is directly related to $I(\boldsymbol{C})$ : for a given $\delta$, the more $I(\boldsymbol{C})$, the less $\bar{\delta}(\boldsymbol{G})$. The expression of the critical value $\bar{\delta}(\boldsymbol{G})$ involves the value of a linear program that can be computed for specific examples.

Figure 3 provides an example of the ambiguous effect of the number of communities. Indeed, from the linear graph $A$ with 5 communities, the addition of a community on the extremity of the line to form graph $B$ implies a decrease in $\bar{\delta}(\boldsymbol{G})$. For a given $\delta$, this is associated with a decrease in $I(\boldsymbol{C})$. However, the further addition of a community to form graph $\boldsymbol{C}$ implies an increase in $\bar{\delta}(\boldsymbol{G})$. 


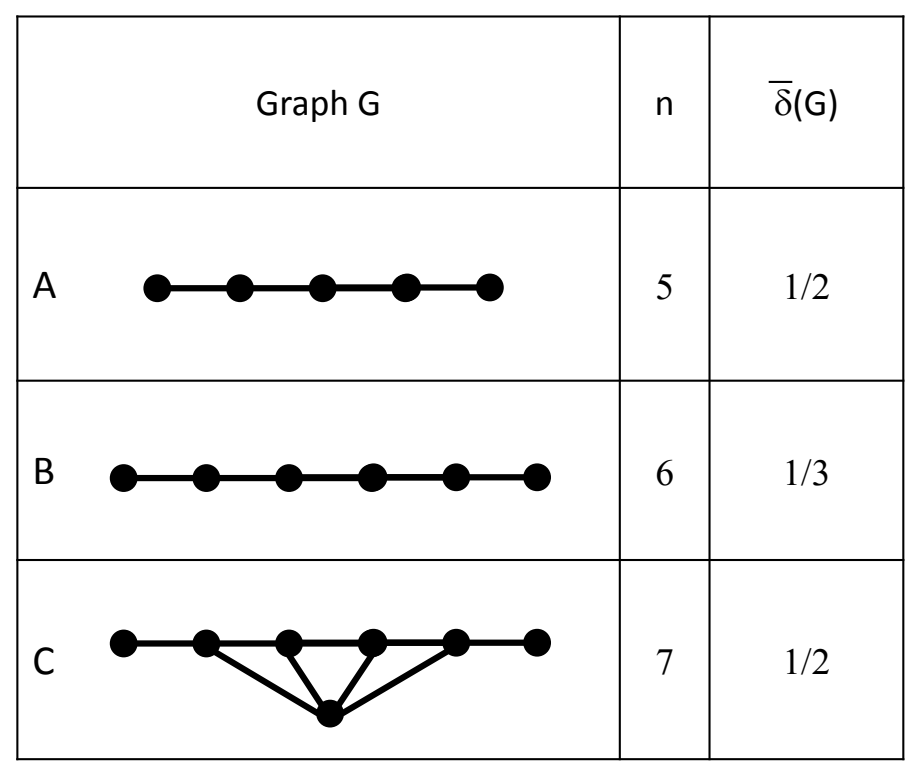

Figure 3: Critical value of $\delta$ for different graphs with different number of communities. These values are obtained from the explicit computation of $\bar{\delta}(\boldsymbol{G})$ according to the expression derived in Appendix G. The code used is provided in Appendix H.1.

Figure 4 presents the critical value $\bar{\delta}(\boldsymbol{G})$ associated with different graphs, all involving 6 communities. Proposition 3 is illustrated on graphs A to F and L to P: we observe that, when a link is added while keeping the minimum degree constant, requirements on $\delta$ can only be relaxed. In particular, the lax condition obtained for the complete graph $S$ can easily be extended to all complete graphs. This further emphasizes that our argument mainly stands for local pollutions. Yet, in this case, since neighborhoods and their complements are no longer asymmetric, Assumptions 1 and 4 cannot be met at the same time. Finally, this assessment shows that the spatial structure is in itself an important source of variability for the set of core agreements. We propose to carry further the exercise on a real administrative unit.

\subsection{A tentative assessment on real geographies}

We now briefly discuss how the least-core value could be estimated in real problems. The exercise is illustrative: we introduce a hypothetical problem in a real administrative geographical division.

More precisely, let's consider a hypothetical negotiation among municipalities for locating and funding a facility in the French département of Haute-Garonne. This project would yield a potentially heterogeneous benefit to each municipality. As Proposition 2 emphasizes, we do not need precise knowledge of the benefits, provided that Assumptions 1 and 3 are met. However, Assumption 2 does not hold in this environment. Hence, we rely on the modification of Proposition 2 established in Appendix C. It allows us to drop this assumption at the cost of a different, more realistic one and a slight modification of the expression of $I(\boldsymbol{C})$. What matters most is the structure of costs. Assume that a facility could only be located at the centroid of each municipality ${ }^{15}$. Assume additionally that, wherever it

\footnotetext{
${ }^{15}$ This exercise emphasizes a limitation in our model: in order to compute the cost matrix $C$, a hypothesis
} 


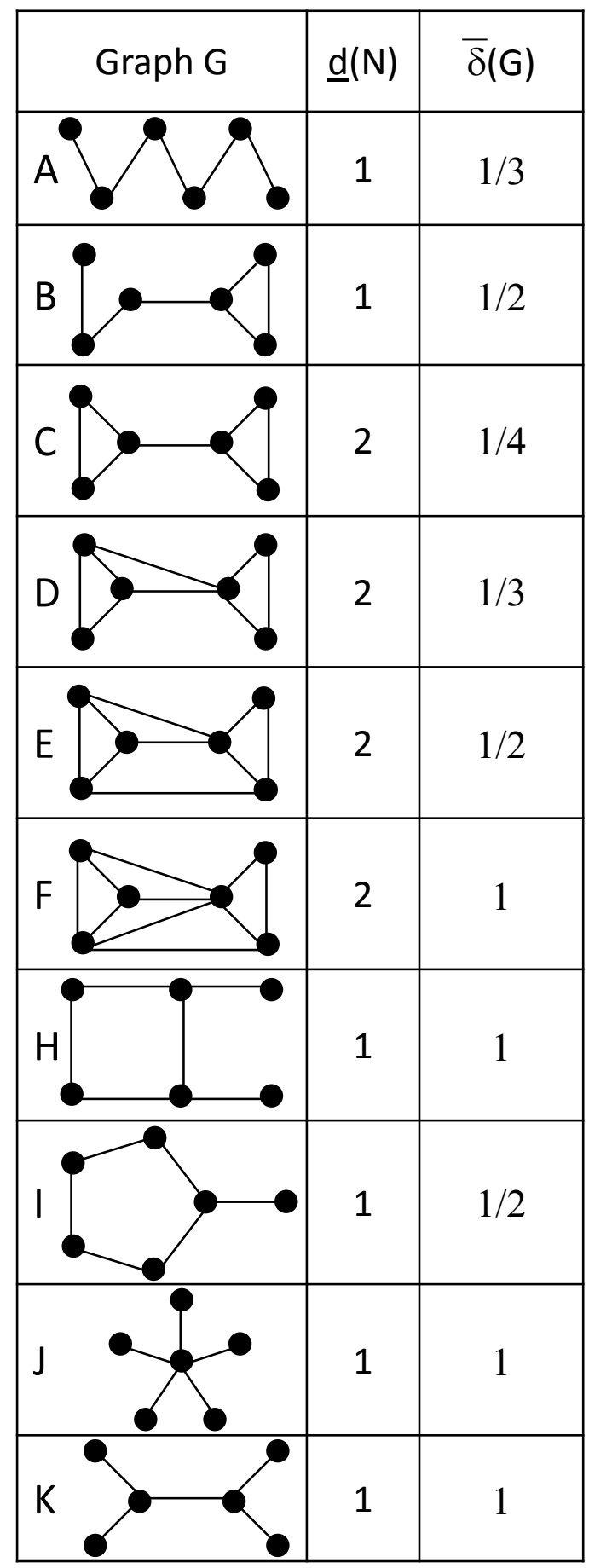

\begin{tabular}{|l|l|l|l|}
\hline & d $(\mathrm{N})$ & $\bar{\delta}(\mathrm{G})$ \\
\hline
\end{tabular}

Figure 4: Critical value of $\delta$ for different graphs with 6 communities where $\underline{d}(N)$ is the minimal degree of the graph. These values are obtained from the explicit computation of $\bar{\delta}(\boldsymbol{G})$ according to the expression derived in Appendix G. The code used is provided in Appendix H.1. 
is built, a facility yields a uniform pollution cost within a fixed radius from the site. For instance, Figure 5 shows, in red, an impacted area of $3 \mathrm{~km}$ around a facility located at the centroid of a given municipality $i$. Given our assumption of uniformity within the impacted area, the total pollution cost is directly proportional to the red area. Moreover, as Proposition 2 emphasizes, we do not need to specify absolute values to compute the index: only relative values matter. Hence, $c_{i i}$ can be normalized to the area of the intersection of the red plain circle and $i$ 's territory, and $c_{i j}$, to the area of the intersection between the red plain circle and $j$ 's territory. The matrix $C$ is obtained by computing all such areas. Neighborhood sets and the optimal location are derived from the matrix $\boldsymbol{C}$.

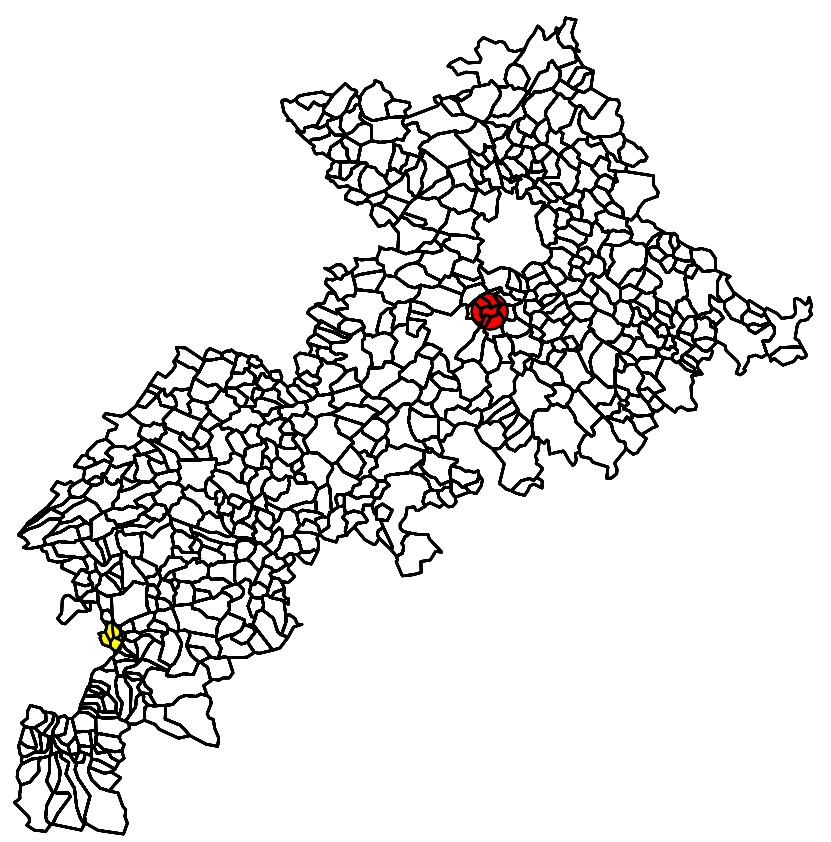

Figure 5: Municipalities composing Haute-Garonne. The red area corresponds to the impacted area when located at an arbitrary municipality for a radius of $3 \mathrm{~km}$. The yellow area corresponds to the impacted area at the optimal location for the same radius.

We compute the least-core value $I(\boldsymbol{C})$ for different radii. The code used to perform this computation is provided in Appendix H.2. It yields the following results.

\begin{tabular}{|c|c|c|c|}
\hline Radius (in km) & 1 & 2 & 3 \\
\hline$I(\boldsymbol{C})$ & 4.46 & 34.2 & 50.2 \\
\hline
\end{tabular}

We note that the least-core value is always higher than 1 , so the core is empty in all these cases. This illustrative exercise can be improved by including a better estimate of the costs of such a facility. The main difficulty here being the use of plausible values for the perceived pollution costs. Any step in this direction would rely on a good understanding of the monetary as well as the non-monetary costs of such facilities. The resulting index would in particular be sensitive to the cost at the optimal site, which, in this illustration, was to be at some indentation of the boundary.

has to be made on where the facility would be located within a given municipality regardless of the coalition it belongs to. In our assessment, we chose the centroids. In a more general framework, we could expect coalitions to have some flexibility in the location choice. By increasing the value of all coalitions, such flexibility would strengthen requirements for non-emptiness. It would yield complications but, in our view, few more insights. 


\section{Conclusion}

In this paper, we analyzed the cooperative provision of economic activities that are globally beneficial but locally harmful in an explicit spatial model. Examples include facilities such as landfills, waste treatment plants or polluting utilities, which require that people living in the neighborhood be compensated. When communities can be excluded from using the facilities, free-riding is not a problem like in standard localized public-good provision problems. In our framework, the exclusion of the neighboring communities is the main obstacle to cooperation. It sets upper bounds on compensations, which together with the participation constraints, determine whether a global cooperative solution exists. That is, if the core of the cooperative game is non-empty. A least-core index is computed to test the existence of a core solution. Its definition is robust to several assumptions on the value function in this cooperative game with externalities. It can be estimated in practice.

As mentioned in the introduction, our investigation of the NIMBY problem using cooperative game theory formalizes bargaining à la Coase in economies with externalities. If, when the core is empty, the parties involved fail to implement the project, the "Coase theorem" does not hold. In the bargaining process, property rights have been assigned to the polluters because a facility could be built without the consent of the neighboring communities. Under other assignments of property rights, the core might be non-empty. In particular, it is easy to show that the core is never empty under the polluter-pays principle: if the communities building the facility are forced to compensate all neighboring communities for the damages, a global and efficient agreement can always be reached. Therefore, in contradiction with another interpretation of Coase's thought, the assignment of property rights could matter for reaching efficiency, even in the absence of transaction costs. 


\section{References}

[1] Varouj A. Aivazian and Jeffrey L. Callen. The coase theorem and the empty core. Journal of Law and Economics, 24(1):175-181, 1981.

[2] Yoram Bachrach, Elkind Edith, Reshef Meir, Dmitrii Pasechnik, Michael Zuckerman, Jorg Rothe, and Jeffrey S. Rosenschein. The cost of stability in coalitional games. In SAGT' 09: 2nd international symposium on algorithmic game theory, pages 122-134, 2009 .

[3] M. Balinski. On the maximum matching set, minimal covering. In Proceedings of the Princeton symposium on mathematical programming, 1970.

[4] Salvador Barberà, Dolors Berga, and Bernardo Moreno. Domains, ranges and strategyproofness: the case of single-dipped preferences. Social Choice and Welfare, 39(2$3): 335-352,2012$.

[5] Theodore Bergstrom, Lawrence Blume, and Hal Varian. On the private provision of public goods. Journal of Public Economics, 29:25-49, 1986.

[6] Francis Bloch. Sequential formation of coalitions in games with externalities and fixed payoff division. Games and Economic Behavior, 14:90-123, 1996.

[7] Parkash Chander and Henry Tulkens. The core of an economy with multilateral environmental externalities. International Journal of Game Theory, 26:379-401, 1997.

[8] Mickael Suk-Young Chwe. Farsighted coalitional stability. Journal of Economic Theory, 63:299-325, 1994.

[9] Ronald Harry Coase. The problem of social cost. Journal of Law and Economics, 3:1, 1960.

[10] Geoffroy De Clippel and Roberto Serrano. Marginal contributions and externalities in the value. Econometrica, 76(6):1413-1436, 2008.

[11] Pierre Dehez. Cooperative provision of indivisible public goods. Theory and Decision, 74:13-29, 2013.

[12] Michel X. Goemans and Martin Skutella. Cooperative facility location games. Journal of Algorithms, 50:194-214, 2004.

[13] Jean-Jacques Laffont. Effets externes et théorie économique. collection Monographies du Séminaire d'Econométrie, 1977.

[14] Jeremi Laurent-Lucchetti and Justin Leroux. Choosing and sharing. Games and Economic Behavior, 73(1):296-300, 2011.

[15] Jeremy Laurent-Lucchetti and Justin Leroux. Lindhal prices solve the nimby problem. Economic Bulletin, 30(3):2457-2463, 2010.

[16] Michel Le Breton and Shlomo Weber. Stability of coalition structures and the principle of optimal partitioning. In Social Choice, Welfare and Ethics, pages 301-319. Cambridge University Press, 1995.

[17] Michel Le Breton and Shlomo Weber. The art of making everybody happy: How to prevent a secession. IMF Staff Papers, 50:p. 403-435, 2003. 
[18] Raul P. Lejano and Climis A. Davos. Siting noxious facilities with victim compensation: n-person games under transferable utility. Socio-Economic Planning Sciences, 35:109-124, 2001.

[19] V Manjunath. Efficient and strategy-proof social choice when preferences are singledipped. Mimeo, 2010.

[20] M. Maschler, B. Peleg, and L. S. Shapley. Geometric properties of the kernel, nucleolus, and related solution concepts. Mathematics and Operations Research, 4(4):303-338, 1979.

[21] Deborah Minehart and Zvika Neeman. Effective siting of waste treatment facilities. Journal of Environmental Economics and Management, 43:303-324, 2002.

[22] David Perez-Castrillo and David Wettstein. Choosing wisely: A multibidding approach. The American Economic Review, 92(5):1577-1587, 2002.

[23] Arthur Cecil Pigou. The Economics of Welfare. 1920.

[24] Debraj Ray and Rajiv Vohra. Equilibrium binding agreements. Journal of Economic Theory, 73:30-78, 1997.

[25] Debraj Ray and Rajiv Vohra. Coalitional power and public goods. Journal of Political Economy, 109(6):1355-1384, 2001.

[26] Barak D. Richman and Christopher Boerner. A transaction cost economizing approach to regulation: Understanding the nimby problem and improving regulatory responses. Yale Journal on Regulation, 23:29-76, 2006.

[27] Toyotaka Sakai. Fair waste pricing: An axiomatic analysis to the nimby problem. Economic Theory, 50(2):499-521, 2012.

[28] Lloyd S. Shapley and Martin Shubik. On the core of an economic system with externalities. The American Economic Review, 4(1):678-684, 1969.

[29] David Starett. A note on externalities and the core. Econometrica, 41(1):179-183, 1973.

[30] Maxwell L. Stearns. The misguided renaissance of social choice. The Yale Law Journal, 103:1219-1292, 1993.

[31] Arthur M. Sullivan. Siting noxious facilities: A siting lottery with victim compensation. Journal of Urban Economics, 31:360-374, 1992.

[32] R. M. Thrall and W. F. Lucas. N-person games in partition function form. Naval Research Logistics Quarterly, 10(1):281-298, 1963.

[33] Sang-Seung Yi. Stable coalition structures with externalities. Games and Economic Behavior, 20:201-237, 1997. 


\section{Appendix}

\section{A Proof of Proposition 1}

Let $\boldsymbol{x}$ be an allocation which meets the conditions stated in Proposition 1, that is, the efficiency condition (1), individual rationality conditions (2), and the following lower bounds for every $S \in \mathcal{Y}=\{N \backslash S \mid S \in \mathcal{N}\} \cup\{N \backslash\{i\} \mid i \in N\}$,

$$
\sum_{i \in S} x_{i} \geq v(S)
$$

We first show that it satisfies the core lower bounds (5) for any arbitrary coalition. Let $T \subseteq N$.

- If $T$ is a non-building coalition, we have $v(T)=0 . \forall i \in T, \boldsymbol{x}$ meets the individual rationality constraint $x_{i} \geq 0$. The sum of these constaints yields Condition (5) for $T$.

- If $T$ is a building coalition, we have $v(T)=b(T)-c(T)$. Let us consider $j^{*} \in$ $\operatorname{argmin}_{j \in T} \sum_{i \in T} c_{i j}$ an optimal site in $T$ and $S^{*}=\stackrel{\circ}{\mathcal{N}}\left(j^{*}\right) \cap(N \backslash T)$, the set of strict neighbors of $j^{*}$ that are not in $T$ and $\bar{T}=N \backslash S^{*}$. Since $\bar{T}=N \backslash S^{*} \in \mathcal{Y}$,

$$
\sum_{i \in \bar{T}} x_{i} \geq b(\bar{T})-c(\bar{T})
$$

Besides, $c(\bar{T}) \leq c(T)$ so:

$$
\sum_{i \in \bar{T}} x_{i} \geq b(\bar{T})-c(T)
$$

For every $i \in N, N \backslash\{i\} \in \mathcal{Y}$ hence $\sum_{j \in N \backslash\{i\}} x_{j} \geq v(N \backslash\{i\})$. This inequality can be rewritten, using the efficiency condition (1), as $x_{i} \leq v(N)-v(N \backslash\{i\})$. We have $\forall i \in N \backslash \mathcal{H}, v(N)-v(N \backslash\{i\}) \leq b_{i}$ and Assumption 2 additionally implies $\forall h \in$ $\mathcal{H}, v(N)-v(N \backslash\{h\}) \leq b_{h}$. Thus, $\forall i \in N,-x_{i} \geq-b_{i}$. From the summation of the latter inequalities for all agents in $\bar{T} \backslash T$ to inequality (6), we obtain $\sum_{i \in T} x_{i} \geq$ $b(T)-c(T)=v(T)$. Hence condition (5) holds for $T$.

We have shown that the core lower bounds can be restricted to coalitions in $\mathcal{Y}$. From Assumption 1, coalitions in $\{N \backslash S \mid S \in \mathcal{N}\}$ are all building coalitions so the constraints associated with them are: $\sum_{i \in N \backslash S} x_{i} \geq b(N \backslash S)-c(N \backslash S)$. Combining them with the efficiency constraints yields conditions (4) in Proposition 1.

\section{B Proof of Proposition 2}

From Proposition 1, the core can be defined as:

$\left\{\boldsymbol{x} \in \mathbb{R}_{+}^{n} \mid \sum_{N} x_{i}=v(N)\right.$ and $\forall S \in \overline{\mathcal{N}}, \sum_{S} x_{i} \leq b(S)-(c(N)-c(N \backslash S))$ and $\left.\forall i \in N, x_{i} \geq 0\right\}$ 
A necessary and sufficient condition for non-emptiness of this set involves the linear program $(L P 1)$ :

$$
\max _{\boldsymbol{x}}\left\{\sum_{i \in N} x_{i} \mid \forall S \in \overline{\mathcal{N}}, \sum_{i \in S} x_{i} \leq b(S)-(c(N)-c(N \backslash S)), \forall i \in N, x_{i} \geq 0\right\} \geq v(N)
$$

Assumption 3 implies that the saving induced by the withdrawal of a community will never overcome the loss of its benefit so for any $S \subseteq T \subseteq N$, we have $v(S) \leq v(T) \leq v(N)$. In particular, for every $S \in \overline{\mathcal{N}}, v(N \backslash S) \leq v(N)$ which implies $b(S)-(c(N)-c(N \backslash S)) \geq 0$. Hence, this linear program is feasible when Assumption 3 is met (take, for all $i \in N, x_{i}=0$ ). Besides, it is bounded (by $\sum_{N} b_{i}$ for instance) so it admits a finite value.

We now show that the individual rationality constraints $x_{i} \geq 0$ are non-binding in $(L P 1)$. We start to show that, for any optimal solution, no community pays more than $\max _{j \in N \backslash\left\{i_{0}\right\}} c_{j i_{0}}$ , the highest external cost it can bear.

Let $\boldsymbol{x}^{*}$ be an optimal solution to $(L P 1)$ and $i_{0} \in N$. Assume that:

$$
\left.x_{i_{0}}^{*}<b_{i_{0}}+\min _{T \in \mathcal{N}: i_{0} \in T}\left(c(N \backslash T)-c\left((N \backslash T) \cup\left\{i_{0}\right\}\right)\right)\right)
$$

We can then increase $x_{i_{0}}^{*}$ by some $\epsilon>0$ such that:

$$
\left.x_{i_{0}}^{*}+\epsilon<b_{i_{0}}+\min _{T \in \overline{\mathcal{N}}: i_{0} \in T}\left(c(N \backslash T)-c\left((N \backslash T) \cup\left\{i_{0}\right\}\right)\right)\right)
$$

Such an increase improves the objective. We shall show that it also leads to a feasible solution. Let $S \in \overline{\mathcal{N}}$ such that $i_{0} \in S$. Because $S \in \overline{\mathcal{N}}, S \backslash\left\{i_{0}\right\}$ also pertains to $\overline{\mathcal{N}}$ (except for the case $S=\left\{i_{0}\right\}$, in which the result is direct). By feasibility of $\boldsymbol{x}^{*}$, we have:

$$
\left.\sum_{i \in S \backslash\left\{i_{0}\right\}} x_{i}^{*} \leq b\left(S \backslash\left\{i_{0}\right\}\right)-\left(c(N)-c\left((N \backslash S) \cup\left\{i_{0}\right\}\right)\right)\right)
$$

Summing inequalities (8) and (9), we get:

$$
\left.\left.\sum_{i \in S} x_{i}^{*}+\epsilon<b(S)-\left(c(N)-c\left((N \backslash S) \cup\left\{i_{0}\right\}\right)\right)\right)+\min _{T \in \mathcal{\mathcal { N }}: i_{0} \in T}\left(c(N \backslash T)-c\left((N \backslash T) \cup\left\{i_{0}\right\}\right)\right)\right)
$$

Since $\left.\left.\min _{T \in \overline{\mathcal{N}}: i_{0} \in T}\left(c(N \backslash T)-c\left((N \backslash T) \cup\left\{i_{0}\right\}\right)\right)\right) \leq c(N \backslash S)-c\left((N \backslash S) \cup\left\{i_{0}\right\}\right)\right)$, we have:

$$
\sum_{i \in S} x_{i}^{*}+\epsilon<b(S)-(c(N)-c(N \backslash S))
$$

All the constraints involving $x_{i_{0}}$ are met. This contradicts the optimality of $\boldsymbol{x}^{*}$. Hence, inequality (7) cannot hold by contradiction. We have:

$$
\left.x_{i_{0}}^{*} \geq b_{i_{0}}+\min _{T \in \overline{\mathcal{N}}: i_{0} \in T}\left(c(N \backslash T)-c\left((N \backslash T) \cup\left\{i_{0}\right\}\right)\right)\right)
$$

Besides, for any $T \in \overline{\mathcal{N}}$ such that $i_{0} \in T$, 


$$
\left.c\left((N \backslash T) \cup\left\{i_{0}\right\}\right)\right)-c(N \backslash T)=\min _{j \in(N \backslash T) \cup\left\{i_{0}\right\}} \sum_{k \in(N \backslash T) \cup\left\{i_{0}\right\}} c_{j k}-\min _{j \in N \backslash T} \sum_{k \in N \backslash T} c_{j k}
$$

Denoting by $j^{*}$ an optimal host in $N \backslash S$, we have:

$$
\left.c\left((N \backslash T) \cup\left\{i_{0}\right\}\right)\right)-c(N \backslash T) \leq \sum_{k \in(N \backslash T) \cup\left\{i_{0}\right\}} c_{j^{*} k}-\sum_{k \in N \backslash T} c_{j^{*} k}=c_{j^{*} i_{0}}
$$

Hence:

$$
\left.c\left((N \backslash T) \cup\left\{i_{0}\right\}\right)\right)-c(N \backslash T) \geq-\max _{j \in N \backslash\left\{i_{0}\right\}} c_{j i_{0}}
$$

From conditions (10) and (11), we get $x_{i_{0}}^{*} \geq b_{i_{0}}-\max _{j \in N \backslash\left\{i_{0}\right\}} c_{j i_{0}}$ and, from Assumption 3, $x_{i_{0}}^{*} \geq 0$. Thus, individual rationality constraints can be discarded from (LP1) without altering the value of the objective. This leads us to consider the linear program (LP2):

$$
\max _{\boldsymbol{x}}\left\{\sum_{i \in N} x_{i} \mid \forall S \in \overline{\mathcal{N}}, \sum_{i \in S} x_{i} \leq b(S)-(c(N)-c(N \backslash S))\right\}
$$

Again, this linear program is bounded and feasible. Therefore, it admits a finite value and so its dual $\left(L P 2^{*}\right)$ :

$$
\min _{\boldsymbol{x}}\left\{\sum_{S \in \overline{\mathcal{N}}} \chi_{S}(b(S)-(c(N)-c(N \backslash S))) \mid \forall i \in N, \sum_{S: i \in S} \chi_{S}=1, \chi_{S} \geq 0\right\}
$$

Which can be further simplified to:

$$
\min _{\boldsymbol{x}}\left\{b(N)-\sum_{S \in \overline{\mathcal{N}}} \chi_{S}(c(N)-c(N \backslash S)) \mid \forall i \in N, \sum_{S: i \in S} \chi_{S}=1, \chi_{S} \geq 0\right\}
$$

A necessary and sufficient condition for non-emptiness of the core is that the value of $\left(L P 2^{*}\right)$ is lower than $v(N)=b(N)-c(N)$. This leads to the following condition:

$$
\max _{\chi}\left\{\sum_{S \in \overline{\mathcal{N}}} \chi_{S}\left(1-\frac{c(N \backslash S)}{c(N)}\right) \mid \forall i \in N, \sum_{S: i \in S} \chi_{S}=1, \chi_{S} \geq 0\right\} \leq 1
$$

\section{Discarding Assumption 2 in Proposition 2}

If $|\mathcal{H}|>1$, the proof of Proposition 2 holds. Here we assume $|\mathcal{H}|=1$ and show that a similar result to Proposition 2 can still be obtained. The difference lies in the fact that the host can get more than $b_{h}$ in core allocations, which prevents an immediate focus on neighborhoods. However, we show that, as soon as an additional assumption is met, requiring that $x_{h} \leq b_{h}$ does not alter the least-core value. This allows a focus on neighborhoods. The proof proceeds as the proof of Propositions 1 and 2: we first discard redundant constraints and simplify non-binding constraints in a linear program related to the emptiness of the core.

Let us denote by $h$ the unique optimal host in $N$ and let $\boldsymbol{x}$ be an allocation which meets the efficiency condition (1), individual rationality constraints (2) and the following core lower bounds for every $S \in \mathcal{Y}^{\prime}=\mathcal{E} \cup \mathcal{E}_{h} \cup\{N \backslash\{i\} \mid i \in N\}$,

$$
\sum_{i \in S} x_{i} \geq v(S)
$$


Where $\mathcal{E}=\{N \backslash S \mid S \in \stackrel{\circ}{\mathcal{N}}$ and $c(N \backslash S) \leq c(N)\}$ and $\mathcal{E}_{h}=\{S \subset N \mid h \notin S\}$.

We first show that it satisfies the core lower bounds (12) for any arbitrary coalition. Let $T \subseteq N$.

- If $T$ is a non-building coalition, we have $v(T)=0 . \forall i \in T, \boldsymbol{x}$ meets the individual rationality constraint $x_{i} \geq 0$. The sum of these constraints yields Condition (12) for $T$.

- If $T$ is a building coalition, we have $v(T)=b(T)-c(T)$. Let us consider $j^{*} \in$ $\operatorname{argmin}_{j \in T} \sum_{i \in T} c_{i j}$ an optimal site in $T$ and $S^{*}=\mathcal{N}\left(j^{*}\right) \cap(N \backslash T)$, the set of strict neighbors of $j^{*}$ that are not in $T$. We define $\bar{T}=N \backslash S^{*}$. If $c\left(N \backslash S^{*}\right) \leq c(N)$, then $\bar{T} \in \mathcal{E} \subset \mathcal{Y}^{\prime}$. If $c\left(N \backslash S^{*}\right)>c(N)$, then it must be that $h$ is not in $N \backslash S^{*}$ hence $\bar{T} \in \mathcal{E}_{h} \subset \mathcal{Y}^{\prime}$. Therefore:

$$
\sum_{i \in \bar{T}} x_{i} \geq b(\bar{T})-c(\bar{T})
$$

Besides, $c(\bar{T})=\min _{j \in \bar{T}} \sum_{k \in \bar{T}} c_{j k} \leq \sum_{k \in \bar{T}} c_{j^{*} k}=\sum_{k \in T} c_{j^{*} k}=c(T)$, where the third equality comes from the fact that communities in $\bar{T} \backslash T$ do not belong to the neighborhood of $j^{*}$ by construction. Hence:

$$
\sum_{i \in \bar{T}} x_{i} \geq b(\bar{T})-c(T)
$$

The rationality of coalitions $N \backslash\{i\}$ yields $\forall i \in N \backslash\{h\},-x_{i} \geq-b_{i}$. From the summation of the latter inequalities for all agents in $\bar{T} \backslash T$ to inequality (13), we obtain $\sum_{i \in T} x_{i} \geq b(T)-c(T)=v(T)$. Hence condition (12) holds for $T$.

We have shown that the core lower bounds can be restricted to coalitions in $\mathcal{Y}^{\prime}$. Combining them with the efficiency constraints and defining $\overline{\mathcal{E}}=\{T \mid T \in \mathcal{N}$ and $c(N \backslash T) \leq c(N)\}$ and $\overline{\mathcal{E}}_{h}=\{T \mid h \in T\}$, the respective complementary of $\mathcal{E}$ and $\mathcal{E}_{h}$, the core is non-empty if and only if:

$\max _{\boldsymbol{x}}\left\{\sum_{i \in N} x_{i} \mid \forall S \in \overline{\mathcal{E}} \cup \overline{\mathcal{E}}_{h} \cup\{i \mid i \in N\}, \sum_{i \in S} x_{i} \leq b(S)-(c(N)-c(N \backslash S)), \forall i \in N, x_{i} \geq 0\right\} \geq v(N)$

We now eliminate constraints in $\overline{\mathcal{E}}_{h}$. Let us denote by $(L P 3)$ the former linear program. Let us consider $\boldsymbol{x}^{*}$ as an optimal solution to $(L P 3)$ and let us assume $x_{h}^{*}>b_{h}$ so that we can write $x_{h}^{*}=b_{h}+\epsilon, \epsilon>0$. At this stage, an additional assumption is required:

Assumption 6. $\exists S \in \stackrel{\circ}{\mathcal{N}}$ such that $h \notin S$ and $c(N \backslash S) \leq c(N)$

This assumption implies that it is always possible to exclude some agents different from $h$ and save on the cost of the project. We will show there always exists another optimal solution, $\boldsymbol{x}^{\prime}$, such that $x_{h}^{\prime} \leq b_{h}$. From Assumption 6, there exists $S \in \mathcal{N}$ such that $h \notin S$ and $c(N \backslash S) \leq c(N)$. Let us consider $S \cup\{h\} \in \overline{\mathcal{E}}_{h}$; we have, by feasibility of $\boldsymbol{x}^{*}$ in (LP3):

$$
\sum_{i \in S} x_{i}^{*}+x_{h}^{*} \leq \sum_{i \in S} b_{i}+b_{h}
$$

Hence, 


$$
\sum_{i \in S} x_{i}^{*} \leq \sum_{i \in S} b_{i}-\epsilon
$$

Besides the rationality of coalitions $N \backslash\{i\}$ requires $\forall i \in S, x_{i}^{*} \leq b_{i}$. Hence, there exists $\left(\epsilon_{i}\right)_{i \in S} \in \mathbb{R}_{+}^{|S|}$ such that, $\sum_{i \in S} \epsilon_{i}=\epsilon$ and, for all $i \in S, x_{i}^{*} \leq b_{i}-\epsilon_{i}$. Let us define $\boldsymbol{x}^{\prime}$ as follows:

$x_{h}^{\prime}=x_{h}^{*}-\epsilon=b_{h}$

$x_{j}^{\prime}=x_{j}^{*}+\epsilon_{i}$ for all $j \in S$

$x_{i}^{\prime}=x_{i}^{*}$ for all $i \notin S \cup\{h\}$

By construction this solution yields the same objective. We want to show it is feasible as well. Let $T_{S}$ be such that $T_{S} \cap S \neq \emptyset$ and $T_{S} \in \overline{\mathcal{E}} \cup \overline{\mathcal{E}}_{h} \cup\{i \mid i \in N\}$, an arbitrary coalition of $\overline{\mathcal{E}} \cup \overline{\mathcal{E}}_{h} \cup\{i \mid i \in N\}$ containing elements of $S$. Three cases arise:

- If $T_{S} \in\{i \mid i \in N\}$, then the associated constraint $x_{i} \leq b_{i}$ is met by construction.

- If $T_{S} \in \overline{\mathcal{E}}_{h}$, we have, where the first inequality comes from the fact that $\sum_{i \in T_{S} \cap S} \epsilon_{i}-$ $\epsilon \leq 0$ and the second is the feasibility of $\boldsymbol{x}^{*}$ in $(L P 4)$ :

$$
\sum_{i \in T_{S}} x_{i}^{\prime} \leq \sum_{i \in T_{S}} x_{i}^{*} \leq b\left(T_{S}\right)-\left(c(N)-c\left(N \backslash T_{S}\right)\right)
$$

- If $T_{S} \in \overline{\mathcal{E}}, T_{S} \cup\{h\} \in \overline{\mathcal{E}}_{h}$ and by feasibility of $\boldsymbol{x}^{*}$ in $(L P 4)$ :

$$
\sum_{i \in T_{S}} x_{i}^{*}+x_{h}^{*} \leq b\left(T_{S}\right)+b_{h}-\left(c(N)-c\left(N \backslash\left(T_{S} \cup\{h\}\right)\right)\right)
$$

Simplifying $b_{h}$ and because $\sum_{i \in T_{S} \cap S} \epsilon_{i} \leq \epsilon$,

$$
\sum_{i \in T_{S}} x_{i}^{\prime} \leq \sum_{i \in T_{S}} x_{i}^{*}+\epsilon \leq b\left(T_{S}\right)-\left(c(N)-c\left(N \backslash\left(T_{S} \cup\{h\}\right)\right)\right)
$$

Because $c\left(N \backslash T_{S}\right) \leq c(N)$, the optimal location in $N \backslash T_{S}$ cannot be $h$. Hence, the withdrawal of $h$ can only lead to a decrease in cost, so that $c\left(N \backslash\left(T_{S} \cup\{h\}\right)\right) \leq c\left(N \backslash T_{S}\right)$.

Finally, we have, for any constraint $T_{S}$ involving elements of $S$ :

$$
\sum_{i \in T_{S}} x_{i}^{\prime} \leq b\left(T_{S}\right)-\left(c(N)-c\left(N \backslash T_{S}\right)\right)
$$

This establishes that $\boldsymbol{x}^{\prime}$ is feasible. Hence it is an optimal solution as well. Finally, we can require that $x_{h} \leq b_{h}$ without altering the value of the linear program. This defines the linear program $(L P 4)$ :

$$
\max _{\boldsymbol{x}}\left\{\sum_{i \in N} x_{i} \mid \forall S \in \overline{\mathcal{E}} \cup \overline{\mathcal{E}}_{h}, \sum_{i \in S} x_{i} \leq b(S)-(c(N)-c(N \backslash S)), \forall i \in N, x_{i} \leq b_{i} \text { and } x_{i} \geq 0\right\}
$$

It is straightforward to show that, following the introduction of the additional constraint $x_{h} \leq b_{h}$, all constraints in $\overline{\mathcal{E}}_{h}$ are redundant in $(L P 4)$. Hence $(L P 4)$ can be rewritten: 


$$
\max _{\boldsymbol{x}}\left\{\sum_{i \in N} x_{i} \mid \forall S \in \overline{\mathcal{E}}, \sum_{i \in S} x_{i} \leq b(S)-(c(N)-c(N \backslash S)), x_{h} \leq b_{h}, \forall i \in N, x_{i} \geq 0\right\}
$$

And, adding some redundant constraints to simplify the notations:

$$
I(\boldsymbol{C})=\max _{\boldsymbol{x}}\left\{\sum_{i \in N} x_{i} \mid \forall S \in \overline{\mathcal{N}}, \sum_{i \in S} x_{i} \leq b(S)-(c(N)-c(N \backslash S)), \forall i \in N, x_{i} \leq b_{i} \text { and } x_{i} \geq 0\right\}
$$

We eventually get an expression similar to the one introduced in Proposition 2: Assumption 2 can be replaced by Assumption 6 provided we impose the additional condition $x_{h} \leq b_{h}$ in the former linear program. Hence, an expression of $I(\boldsymbol{C})$ can be obtained by defining the function $c^{\prime}$ such that $c^{\prime}(N \backslash\{h\})=0$ and, for all $S \subset N$ different from $N \backslash\{h\}$, $c^{\prime}(S)=c(S)$. Then:

$$
I(\boldsymbol{C})=\max _{\chi}\left\{\sum_{S \in \overline{\mathcal{N}}} \chi_{S}\left(1-\frac{c^{\prime}(N \backslash S)}{c^{\prime}(N)}\right) \mid \forall i \in N, \sum_{S: i \in S} \chi_{S}=1, \chi_{S} \geq 0\right\}
$$

\section{Proof of Corollary 1}

In the linear case, Assumption 2 holds, and we explicitly compute the value of $I(\boldsymbol{C})$. In this section we will use the notion of balanced collections. A collection $\mathcal{B}$ of subsets of $N$ is said to be balanced if and only if there exist strictly positive weights $\chi^{\mathcal{B}}=\left(\chi_{S}^{\mathcal{B}}\right)_{S \in \mathcal{B}}$ such that, for any $i \in N, \sum_{S \in \overline{\mathcal{N}}: i \in S} \chi_{S}^{\mathcal{B}}=1$. Denoting by $\mathbb{B}(\overline{\mathcal{N}})$ the set of balanced collections over $N$ composed of elements of $\overline{\mathcal{N}}$ only, we can write:

$$
I(\boldsymbol{C})=\frac{1}{c(N)} \max _{\mathcal{B} \in \mathbb{B}(\mathcal{N})}\left\{\sum_{S \in \mathcal{B}} \chi_{S}^{\mathcal{B}}(c(N)-c(N \backslash S))\right\}
$$

For any $S \in \mathcal{N}$, we have the corresponding values:

$$
c(N)-c(N \backslash S)=\left\{\begin{array}{l}
\delta c \text { if } S \in\{2, n-1\} \\
\delta c \text { if } S \in\{j \cup j+2 \mid j \in[1, n-2]\} \\
0 \text { otherwise }
\end{array}\right.
$$

$\overline{\mathcal{N}}$ is a set of coalitions of no more than two players. Hence, for any balanced collection $\mathcal{B}$ of elements of $\overline{\mathcal{N}}$, there exists a partition of $N$ into pairwise disjoint sets $N_{1}, \ldots, N_{l}, l=0 \ldots L$ where each $N_{l}$ with $l>0$ is a coalition of at least three communities such that $\mathcal{B}$ consists of full cycles on each $N_{l}$ and a partition of $N_{0}$ (Balinski, 1970, as stated in Le Breton and Weber, 1995: 316). Because no cycle can be formed out of elements of $\overline{\mathcal{N}}$ in the linear case, all balanced coalitions over $\overline{\mathcal{N}}$ are partitions. In summary, we are interested in finding partitions $\mathcal{P}$ of $N$, composed with elements of $\overline{\mathcal{N}}$ which maximize $\sum_{S \in \mathcal{P}}(c(N)-c(N \backslash S))$. We now explain how to find such optimal partitions.

First, for any partition involving coalitions in which 2 or $n-1$ belongs to a two-agent coalition, we weakly improve on the objective by splitting such coalitions into singletons. Hence, we can restrict our attention to coalitions in which such communities appear as singletons. The construction of an optimal partition then consists in maximizing the 
number of coalitions of the form $\{j \cup j+2 \mid j \in[1, n-2]\}$. In the case $n \in\{4,5,6,7\}$, such optimal partitions are trivial as soon as communities 2 and $n-1$ appear as singletons. Figure 6 presents optimal partitions and the corresponding value of $\sum_{S \in \mathcal{P}}(c(N)-c(N \backslash S))$.

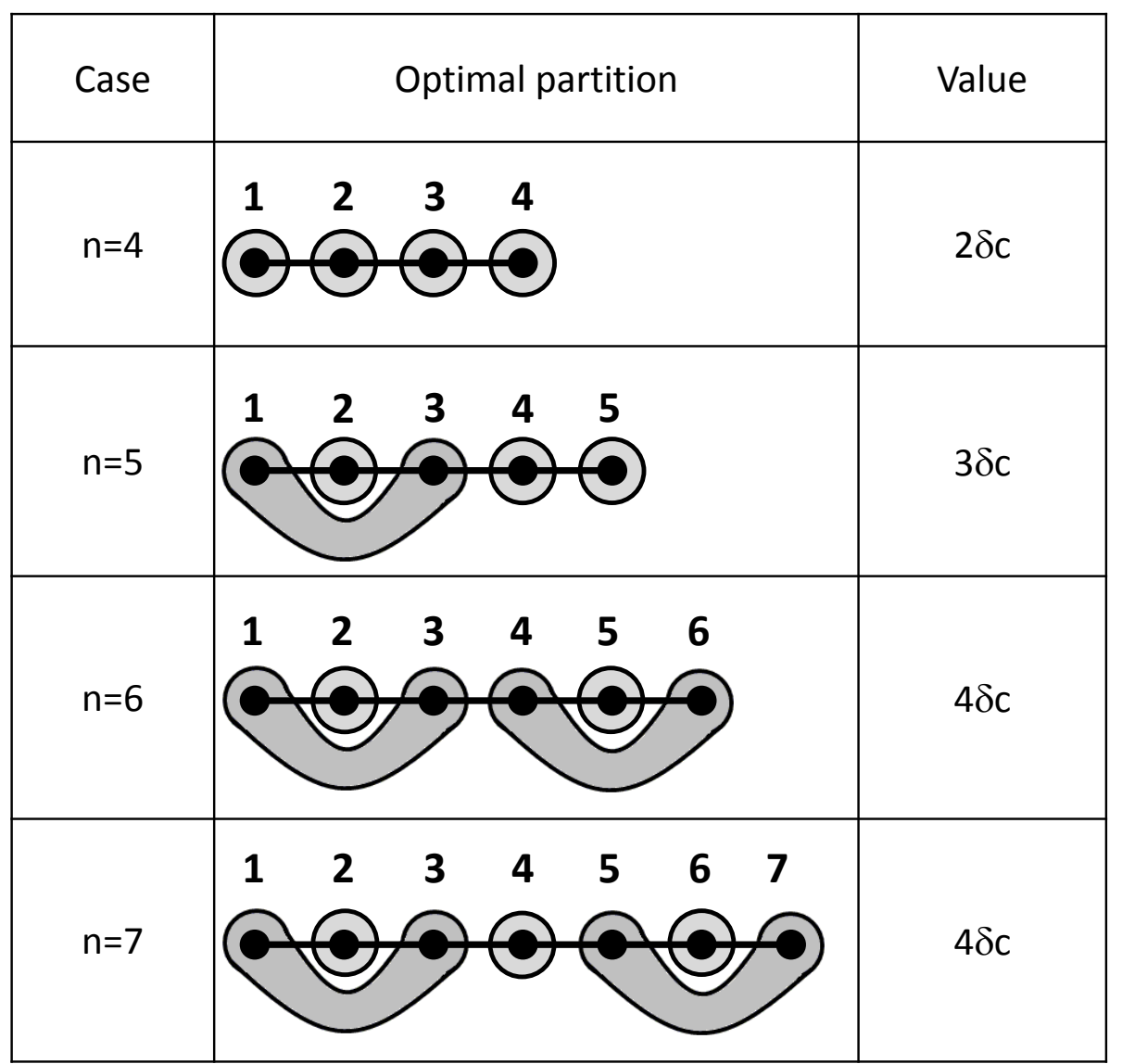

Figure 6: Initial patterns. The reasoning adopted for finding the optimal partitions consists in considering all possible cases. We detail the case $n=7$. First, we know that there is always an optimal partition containing $\{2\}$ and $\{6\}$ as singletons. The value associated with each is $\delta c$. The value associated with any other single individual is 0 whereas the value associated with any pair of $\overline{\mathcal{N}}$ is $\delta c$. An optimal partition thus contains as many pairs of $\overline{\mathcal{N}}$ as possible. This is achieved with the partition $\mathcal{P}=\{\{1,3\},\{2\},\{4\},\{6\},\{5,7\}\}$.

For any $n>7$, we know that $n$ can be decomposed as $n=4 k+i, k \in \mathbb{N}$ and $i \in 0,1,2,3$. According to this decomposition, an optimal partition can be found by combining the initial patterns above and the iterative pattern presented in Figure 7 which maximizes the value that can be obtained by adding 4 communities to the initial pattern. 


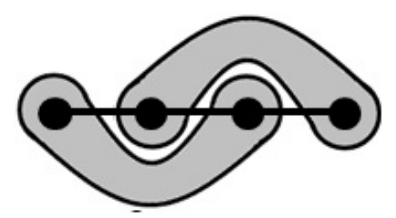

Figure 7: Iterative pattern

We eventually find the following optimal partitions:

- If $n=4 k, k \in \mathbb{N}, \mathcal{P}=\{\{1\},\{2\},\{n-1\},\{n\}\} \cup_{j=1}^{k-1}\{\{4 j-1,4 j+1\},\{4 j, 4 j+2\}\}$

- If $n=4 k+1, k \in \mathbb{N}, \mathcal{P}=\{\{1,3\},\{2\},\{n-1\},\{n\}\} \cup_{j=1}^{k-1}\{\{4 j, 4 j+2\},\{4 j+1,4 j+3\}\}$

- If $n=4 k+2, k \in \mathbb{N}, \mathcal{P}=\{\{1,3\},\{2\},\{n-1\},\{n-2, n\}\} \cup_{j=1}^{k-1}\{\{4 j, 4 j+2\},\{4 j+$ $1,4 j+3\}\}$

- If $n=4 k+3, k \in \mathbb{N}, \mathcal{P}=\{\{1,3\},\{2\},\{4\},\{n-1\},\{n-2, n\}\} \cup_{j=1}^{k-1}\{\{4 j+1,4 j+$ $3\},\{4 j+2,4 j+4\}\}$

And the associated values are:

$$
I(\boldsymbol{C})=\left\{\begin{array}{l}
\frac{n}{2} \frac{\delta}{1+\delta} \text { if } n=4 k, k \in \mathbb{N} \\
\frac{n+1}{2} \frac{\delta}{1+\delta} \text { if } n=4 k+1, k \in \mathbb{N} \\
\frac{n+2}{2} \frac{\delta}{1+\delta} \text { if } n=4 k+2, k \in \mathbb{N} \\
\frac{n+1}{2} \frac{\delta}{1+\delta} \text { if } n=4 k+3, k \in \mathbb{N}
\end{array}\right.
$$

The condition on $\delta$ expressed in Corollary 1 directly follows from the comparison of $I(\boldsymbol{C})$ with 1 .

\section{E Proof of Proposition 3}

Let $\sigma=\left(N, \boldsymbol{b}, \boldsymbol{C}=\left(c_{i j}\right)_{(i, j) \in N^{2}}\right)$ and $\sigma^{+}=\left(N, \boldsymbol{b}, \boldsymbol{C}^{+}=\left(c_{i j}^{+}\right)_{(i, j) \in N^{2}}\right)$ be two NIMBY problems meeting Assumptions 1 and 2. Let us respectively define $c$ and $v$ (respectively $c^{+}$and $v^{+}$) the cost and the value function in the problem $\sigma$ (resp. $\sigma^{+}$). We assume

1. $c(N)=c^{+}(N)$;

2. $\forall(i, j) \in N^{2}, c_{i j} \leq c_{i j}^{+}$. We will note $\boldsymbol{C} \leq \boldsymbol{C}^{+}$.

Let $\left(L P 3^{*}\right)$ and $\left(L P 3_{+}^{*}\right)$ be the linear programs defining respectively $I(C)$ and $I\left(C^{+}\right)$ and let $\chi^{*}$ be an optimal solution to $\left(L P 3^{*}\right) . \chi^{*}$ is feasible in $\left(L P 3_{+}^{*}\right)$. Besides, because $\boldsymbol{C} \leq \boldsymbol{C}^{+}$and $c(N)=c^{+}(N)$, we have $\forall S \in \mathcal{N},\left(1-\frac{c(N \backslash S)}{c(N)}\right) \geq\left(1-\frac{c(N \backslash S)}{c(N)}\right)$, so the value of the linear program $\left(L P 3_{+}^{*}\right)$ taken at $\chi^{*}$ is not lower than $I(C)$. Therefore, its optimal value $I\left(\boldsymbol{C}^{+}\right)$cannot be lower than $I(\boldsymbol{C})$. 


\section{F Proof of Proposition 5}

Let $R$ be a restriction and $v^{R}$ its associated characteristic function. We want to show that under Assumptions 1, 2, 4 and 5, the $R$-core is non-empty if and only if $I(\boldsymbol{C}) \geq 1$. We extend the proof of Propositions 1 and 2.

First, we eliminate redundant constraints in the system defining the core. We distinguish between building and non-building coalitions. $N B=\{T \subset N \mid b(T)<c(T)\}$ is the set of non-building coalitions. Replicating the proof of Proposition 1, the constraints for building coalitions can be restricted to $\{N \backslash S \mid S \in \overline{\mathcal{N}}\}$. However, the constraints for non-building coalitions cannot be reduced to individual rationality: an allocation $\boldsymbol{x}$ is in the $R$-core $\mathcal{C}^{R}$ if and only if

$$
\begin{gathered}
\sum_{i \in N} x_{i}=v(N) \\
\forall S \in N B, \sum_{i \in S} x_{i} \geq v^{R}(S) \\
\forall i \in N, x_{i} \geq b_{i} \\
\forall S \in \stackrel{\circ}{\mathcal{N}}, \sum_{i \in S} x_{i} \leq b(S)-(c(N)-c(N \backslash S))
\end{gathered}
$$

where the constraints (15) contain the individual rationality constraints. We consider the linear program $(L P 5)$ :

$$
\max _{\boldsymbol{x}}\left\{\sum_{i \in N} x_{i} \mid \forall S \in \overline{\mathcal{N}}, \sum_{i \in S} x_{i} \leq b(S)-(c(N)-c(N \backslash S)) \text { and } \forall S \in N B, \sum_{i \in S} x_{i} \geq v^{R}(S)\right\}
$$

The $R$-core $\mathcal{C}^{R}$ is non-empty if and only if $(L P 4)$ is feasible and reaches a value higher than $v(N)$. We first note that such a program would always be feasible under Assumption 5 (e.g. $\boldsymbol{x}$ such that $\forall i \in N, x_{i}=b_{i}-\max _{j \in N \backslash\{i\}} c_{j i}$ is feasible). Second, as in the proof of Proposition 2, we can show that the constraints on the right are never binding under Assumption 5.

Let $\boldsymbol{x}^{*}$ be an optimal solution to the above linear program and $i_{0} \in N$. Assume:

$$
\left.x_{i_{0}}^{*}<b_{i_{0}}+\min _{T \in \overline{\mathcal{N}}: i_{0} \in T}\left(c(N \backslash T)-c\left((N \backslash T) \cup\left\{i_{0}\right\}\right)\right)\right)
$$

Then we can increase $x_{i_{0}}^{*}$ by some $\epsilon>0$ such that:

$$
\left.x_{i_{0}}^{*}+\epsilon<b_{i_{0}}+\min _{T \in \mathcal{N}: i_{0} \in T}\left(c(N \backslash T)-c\left((N \backslash T) \cup\left\{i_{0}\right\}\right)\right)\right)
$$

Such an increase improves on the objective. We shall show that it also leads to a feasible solution.

Let $S \in \overline{\mathcal{N}}$ with at least two communities, such that $i_{0} \in S$. Because $S \in \overline{\mathcal{N}}, S \backslash\left\{i_{0}\right\}$ also pertains to $\overline{\mathcal{N}}$. By feasibility of $\boldsymbol{x}^{*}$, we have: 


$$
\left.\sum_{i \in S \backslash\left\{i_{0}\right\}} x_{i}^{*} \leq b\left(S \backslash\left\{i_{0}\right\}\right)-\left(c(N)-c\left((N \backslash S) \cup\left\{i_{0}\right\}\right)\right)\right)
$$

Summing inequalities (19) and (20), we get:

$$
\left.\left.\sum_{i \in S} x_{i}^{*}+\epsilon<b(S)-\left(c(N)-c\left((N \backslash S) \cup\left\{i_{0}\right\}\right)\right)\right)+\min _{T \in \mathcal{\mathcal { N }}: i_{0} \in T}\left(c(N \backslash T)-c\left((N \backslash T) \cup\left\{i_{0}\right\}\right)\right)\right)
$$

Therefore,

$$
\sum_{i \in S} x_{i}^{*}+\epsilon<b(S)-(c(N)-c(N \backslash S))
$$

In this case, we additionally have to show that constraints in $N B$ are met, which is straightforward. All the constraints involving $x_{i_{0}}$ are met. This contradicts the optimality of $\boldsymbol{x}^{*}$. Hence, inequality (18) cannot hold by contradiction. We have:

$$
\left.x_{i_{0}}^{*} \geq b_{i_{0}}+\min _{T \in \overline{\mathcal{N}}: i_{0} \in T}\left(c(N \backslash T)-c\left((N \backslash T) \cup\left\{i_{0}\right\}\right)\right)\right)
$$

Besides, as established in the proof of Proposition 2:

$$
\left.\forall S \in \overline{\mathcal{N}}: i_{0} \in S, c(N \backslash S)-c\left((N \backslash S) \cup\left\{i_{0}\right\}\right)\right) \geq-\max _{j \in N \backslash\left\{i_{0}\right\}} c_{j i_{0}}
$$

so $x_{i_{0}}^{*} \geq b_{i_{0}}-\max _{j \in N \backslash\left\{i_{0}\right\}} c_{j i_{0}}$ and $\forall S \in\{T \subset N \mid b(T)<c(T)\}, \sum_{i \in T} x_{i} \geq b(T)-$ $\sum_{i \in T} \max _{j \in N \backslash\{i\}} c_{j i}$. Hence, using Assumption 5, $\sum_{i \in T} x_{i} \geq v^{R}(T)$.

All constraints for coalitions in $N B$ can then be removed from the program without changing the value of the objective. This leads us back to the linear program (LP2) and the proof of Proposition 2 applies.

\section{G Proof of Corollary 2}

The cost of the project on a graph depends on the minimal degree of this graph. For any $S \subseteq N$, we denote by $\underline{d}(S)$ the minimal degree of the graph induced by $S$ on $G$. Rewriting the condition $I(\boldsymbol{C}) \geq 1$, we get the following condition on $\delta$ :

$$
\delta \leq \bar{\delta}(\boldsymbol{G})=\frac{1}{\max \chi\left\{\sum_{S \in \overline{\mathcal{N}}} \chi_{S}(\underline{d}(N)-\underline{d}(N \backslash S)) \mid \forall i \in N, \sum_{S: i \in S} \chi_{S}=1, \chi_{S} \geq 0\right\}-\underline{d}(N)}
$$

We want to show $\bar{\delta}(\boldsymbol{G})>0$. Let $h \in \mathcal{H}$ be an optimal host in $N$ and $j \in \stackrel{\circ}{\mathcal{N}}(h)^{16}$. Consider the following partition: $\left\{\stackrel{\circ}{\mathcal{N}}(h), S_{j}, N \backslash\left(\stackrel{\circ}{\mathcal{N}}(h) \cup S_{j}\right)\right\}$, where $S_{j}=\stackrel{\circ}{\mathcal{N}}(j) \backslash \stackrel{\circ}{\mathcal{N}}(h)$ is the strict neighborhood $j$ from which we withdraw members of $\stackrel{\circ}{\mathcal{N}}(h)$. A feasible solution $\chi^{\prime}$ associated with this partition is defined as follows:

\footnotetext{
${ }^{16}$ We assume here that $|\stackrel{\mathcal{N}}{ }(h)|>0$. If it is not, the core is always non-empty $(\bar{\delta}(\boldsymbol{G})=+\infty)$.
} 
- $\chi_{\mathcal{N}(h)}^{\prime}=1$

- $\chi_{S_{j}}^{\prime}=1$;

- $\chi_{N \backslash\left(\mathcal{N}(h) \cup S_{j}\right)}^{\prime}=1$;

- $\chi_{S}^{\prime}=0$ for all other coalitions

We compute the value of this linear program at this feasible solution. First, we know that $|\stackrel{\mathcal{N}}{ }(h)|=\underline{d}(N)$. Hence community $j$ has at most $\underline{d}(N)-1$ neighbors in $\stackrel{\circ}{\mathcal{N}}(h)$. The withdrawal of its neighbors in $S_{j}$ therefore leads to a graph with a degree of at least $\underline{d}(N)-1$. Hence, $\underline{d}(N)-1 \geq \underline{d}\left(N \backslash S_{h}\right)$, which implies that $\underline{d}(N)-\underline{d}\left(N \backslash S_{h}\right) \geq 1$. Second, we have $\underline{d}(N \backslash \stackrel{\circ}{\mathcal{N}}(h))=0$; hence, $\underline{d}(N)-\underline{d}(N \backslash \stackrel{\circ}{\mathcal{N}}(h))=\underline{d}(N)$. Finally, as we have $h \in S_{j}$ by construction, the minimal degree of $\stackrel{\circ}{\mathcal{N}}(h) \cup S_{h}$ is at most $\underline{d}(N)$; hence $\underline{d}(N)-\underline{d}\left(\stackrel{\circ}{\mathcal{N}}(h) \cup S_{h}\right) \geq 0$. The value associated with the feasible solution $\chi^{\prime}$ is $\underline{d}(N)+1$, hence the optimal value of the linear program defining $\bar{\delta}(\boldsymbol{G})$ can only be higher than it. Therefore, $\bar{\delta}(\boldsymbol{G})>0$.

\section{H Code (software R)}

\section{H.1 NIMBY problems of graphs}

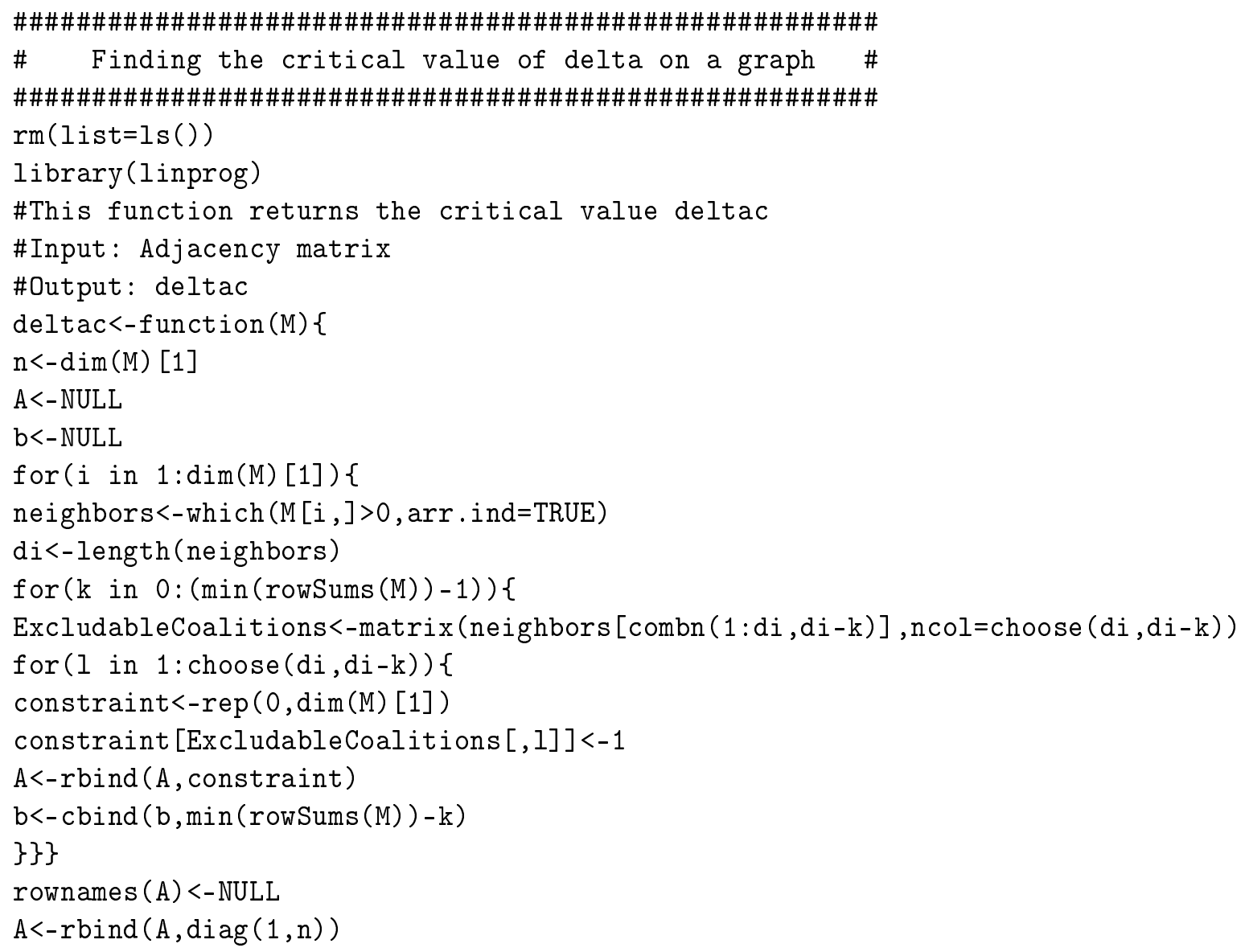




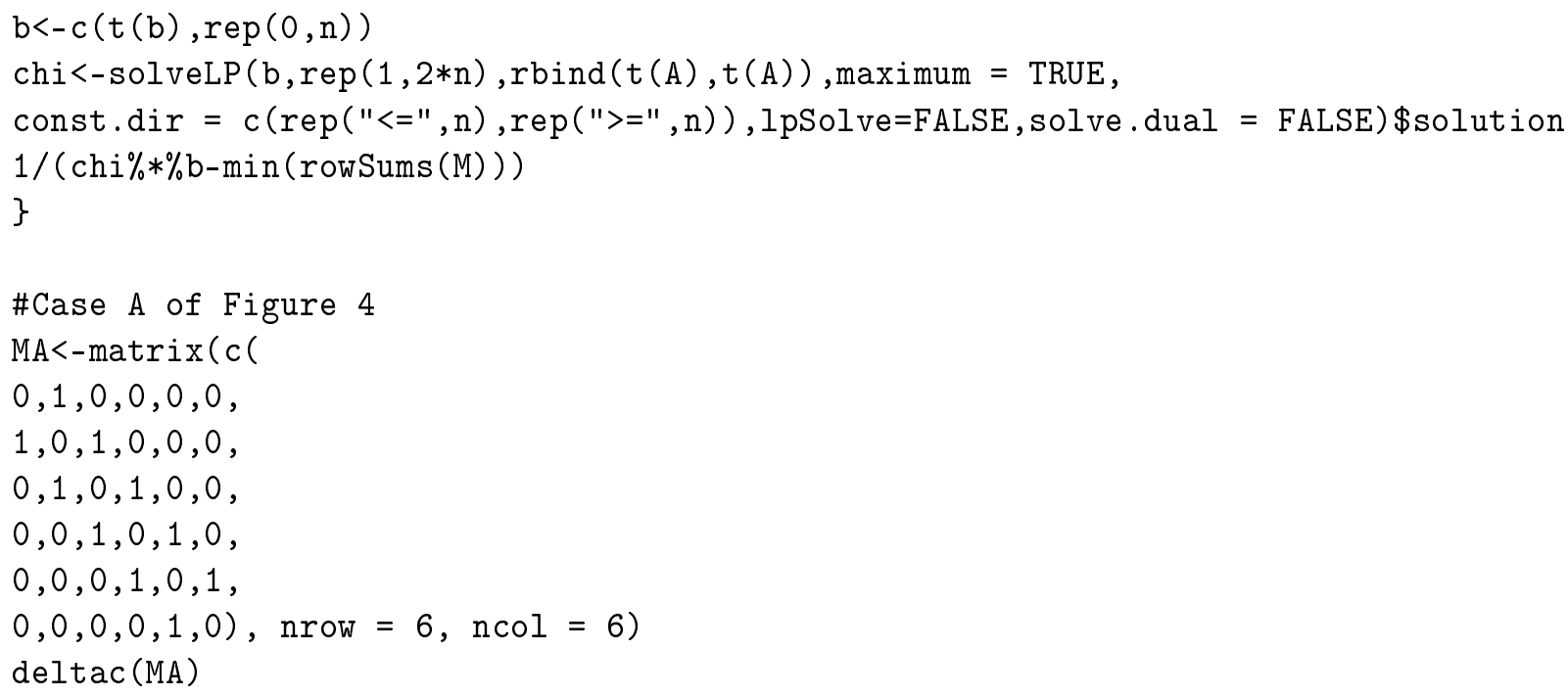

\section{H.2 NIMBY problem on a French administrative unit}

The GIS data used is the GEOFLA@ Communes database. It is publicly available at http://professionnels.ign.fr/geofla.

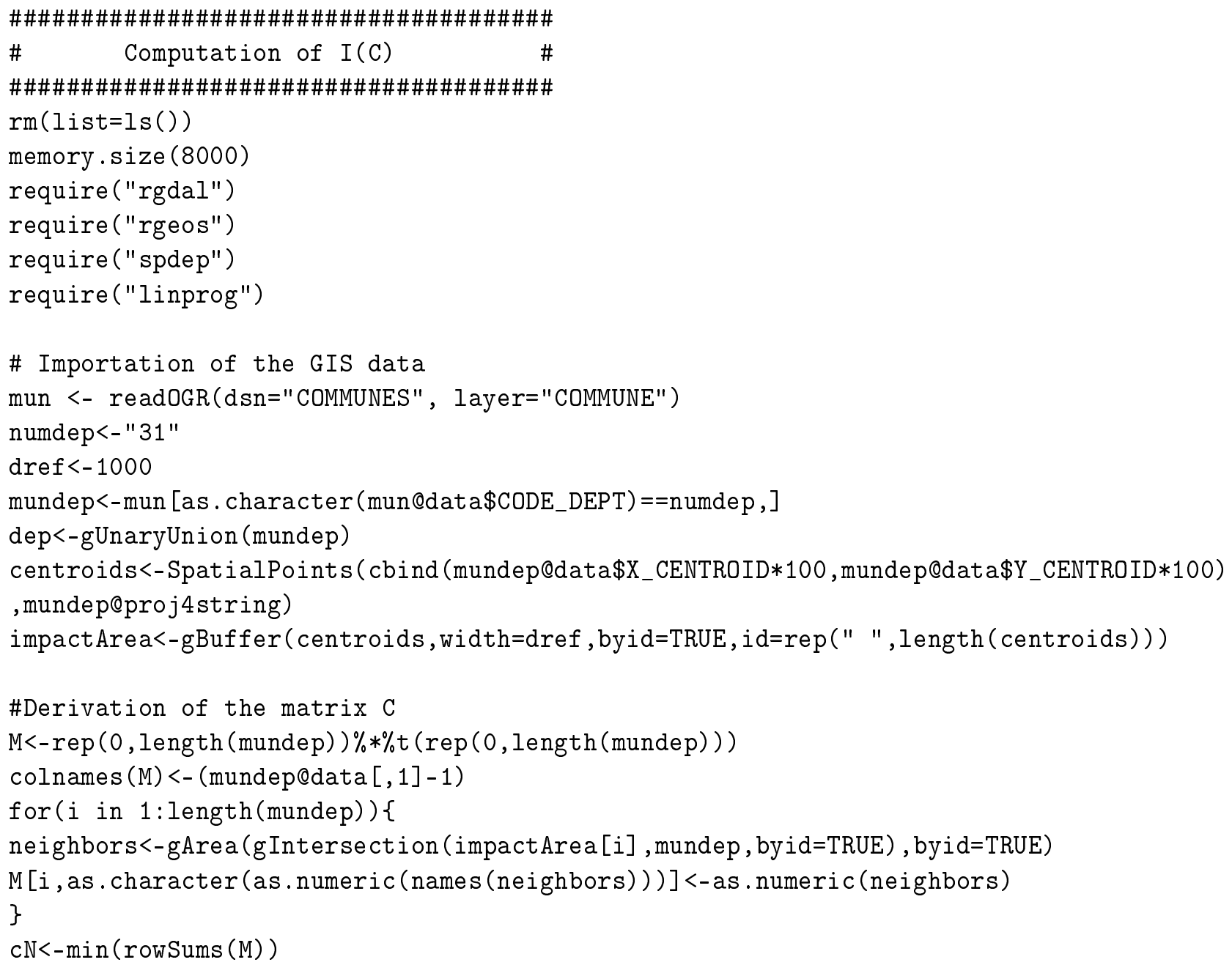




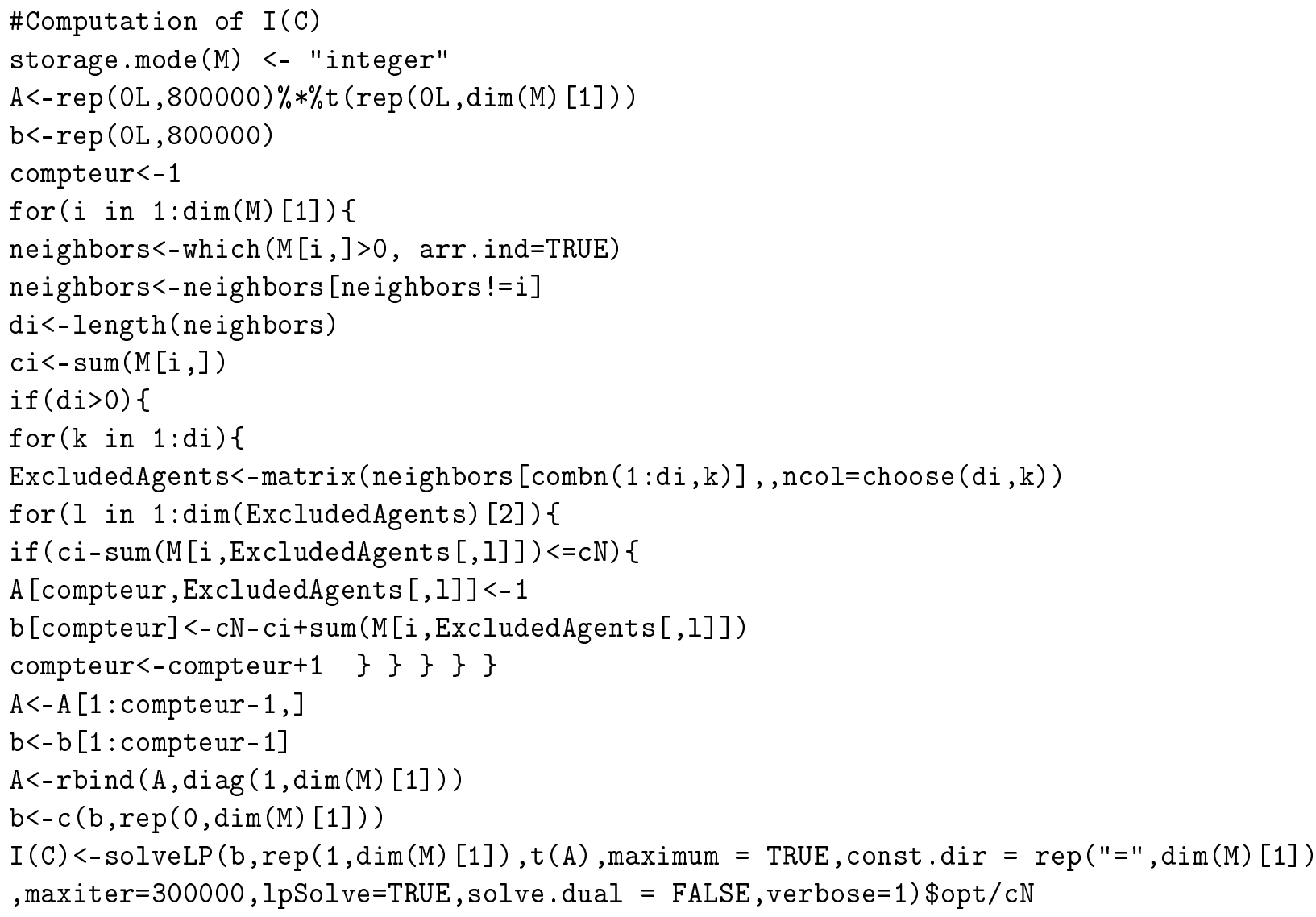

